\title{
COMPLEX GEOMETRY AND THE ASYMPTOTICS OF HARISH-CHANDRA MODULES FOR REAL REDUCTIVE LIE GROUPS. I
}

\author{
LUIS G. CASIAN AND DAVID H. COLLINGWOOD
}

\begin{abstract}
Let $G$ be a connected semisimple real matrix group. It is now apparent that the representation theory of $G$ is intimately connected with the complex geometry of the flag variety $\mathscr{B}$. By studying appropriate orbit structures on $\mathscr{B}$, we are naturally led to representation theory in the category of Harish-Chandra modules $\mathscr{H} \mathscr{C}$, or the representation theory of category $\mathcal{O}^{\prime}$. The Jacquet functor $J$ : $\mathscr{H} \mathscr{C} \rightarrow \mathcal{O}^{\prime}$ has proved a useful tool in converting " $\mathscr{H} \mathscr{C}$ problems" into " $\mathcal{O}$ ' problems," which are of ten more tractable. In this paper, we advance the philosophy that the complex geometry of $\mathscr{B}$, associated to $\mathscr{H} \mathscr{C}$ and $\mathcal{O}^{\prime}$, interacts in a natural way with the functor $J$, leading to deep new information on the structure of Jacquet modules. This, in turn, gives new insight into the structure of certain nilpotent cohomology groups associated to Harish-Chandra modules. Our techniques are based upon many of the ideas present in the proof of the Kazhdan-Lusztig conjectures and Bernstein's proof of the Jantzen conjecture.
\end{abstract}

1. Introduction. Let $G_{\mathbf{R}}$ be a connected semisimple real matrix group. Through the pioneering work of several people, it has now become apparent that the representation theory of $G_{\mathbf{R}}$ is intimately connected with the complex geometry of the flag variety $\mathscr{B}$. By studing appropriate orbit structures on $\mathscr{B}$, we are naturally led to representation theory in the category of Harish-Chandra modules $\mathscr{H} \mathscr{C}$, or the representation theory of category $\mathcal{O}^{\prime}$. As a testament to this approach, the KazhdanLusztig conjectures for $\mathscr{H} \mathscr{C}$ and $\mathcal{O}^{\prime}$ have now been proved. On the other hand, holding to a more classical mindset, we have the Jacquet functor $J: \mathscr{H} \mathscr{C} \rightarrow \mathcal{O}^{\prime}$. This functor has proved a useful tool in converting " $\mathscr{H} \mathscr{C}$ problems" into " $\mathcal{O}^{\prime}$ problems," which are often more tractable. Unfortunately, the full potential of this "transference philosophy" has yet to be fully realized, mainly due to the notoriously obscure $\mathfrak{g}$-module structure of Jacquet modules $J(X), X \in \mathscr{H} \mathscr{C}$. In this paper, we advance the philosophy that the complex geometry of $\mathscr{B}$, associated to $\mathscr{H} \mathscr{C}$ and $\mathcal{O}^{\prime}$, interacts in a natural way with the functor $J$, leading to new information on the structure of Jacquet modules. Our starting point is the fundamental concept of a geometric Jacquet functor, denoted $J_{\text {geo }}$. Such a functor encapsulates the essential properties of the usual Jacquet functor, but naturally extends to a Hecke algebra map between the Hecke modules attached to $\mathscr{H} \mathscr{C}$ and $\mathcal{O}^{\prime}$. (This is just a combinatorial way of saying that $J$ "intertwines" the $\mathscr{B}$ geometry attached to $\mathscr{H} \mathscr{C}$ and $\mathcal{O}^{\prime}$.) Given any irreducible Harish-Chandra module $\bar{\pi}$, we have a natural $g$-filtration of

Received by the editors November 19, 1985 .

1980 Mathematics Subject Classification (1985 Revision). Primary 22E45; Secondary 20G05.

The second author was supported by an NSF Postdoctoral Research Fellowship \# DMS 85-11467. 
$J_{\text {geo }}(\bar{\pi})$ by weights (of Frobenius), which we conjecture to correspond to a g-filtration of $J(\bar{\pi})$ having semisimple subquotients. ${ }^{1}$

Our main results are threefold: (i) we establish the computability of a weight filtration on $J_{\text {geo }}(\bar{\pi})$ and show it is independent of the geometric Jacquet functor chosen; (ii) we establish the existence of a geometric Jacquet functor; and (iii) we verify the conjectural correspondence in a large number of cases (cf. footnote 1). Our work relies heavily on the work of Lusztig-Vogan and Vogan on the Kazhdan-Lusztig conjectures, together with results of Beilinson, Bernstein, Deligne, and Gabber on perverse sheaves and the philosophy of "going to positive characteristic." The paper concludes by remarking on an application to the computation of higher n-homology groups.

In more detail, we fix, once and for all, a reductive affine algebraic group $G$ over $\mathbf{R}$ ( $\mathbf{R}$ the field of real numbers). We look at $G$ as the set of zeros in $\operatorname{GL}(n, \mathbf{C})$ of a finite set of polynomials in the matrix entries with real coefficients. Assume $G_{\mathbf{R}}$ has finite index in the set of real points of $G$. Then $G_{\mathbf{R}}$ is a "real reductive group," in the sense of [36]. Fix a maximal compact subgroup $K_{\mathbf{R}}$ with complexification $K$. Let $B=H_{m} N$ be an "Iwasawa Borel subgroup" of $G$, with $N$ the nilradical and $H_{m, \mathbf{R}}$ a maximally split $\theta$-stable Cartan subgroup; $\theta$ is the Cartan involution attached to $K$.

Denoting by $g$ the complexified Lie algebra of $G_{\mathbf{R}}$, we define $\mathscr{B}$ to be the variety of all Borel subalgebras of $\mathrm{g}$ (the usual flag variety). The groups $K$ and $N$ both act on $\mathscr{B}$ in a natural way, with finitely many orbits. Fix, once and for all, a finite-dimensional irreducible representation $F$ of $G_{\mathbf{R}}$. We introduce $\mathscr{H}_{\mathscr{C}_{F}}$ and $\mathscr{O}_{F}^{\prime}$ to consist of modules in $\mathscr{H} \mathscr{C}$ and $\mathscr{O}^{\prime}$ with the same infinitesimal character as $F$; here $\mathcal{O}^{\prime}$ consists of the $\mathfrak{b}$-finite finitely generated $\mathfrak{U}(\mathrm{g})$-modules. Let $H=K$ or $N$ and define

$$
\mathscr{D}_{H}=\left\{\begin{array}{ll}
(\gamma, \mathcal{O}): & \mathcal{O} \text { is an } H \text {-orbit on } \mathscr{B} \text { and } \gamma \text { is } \\
& \text { an } H \text {-homogeneous line bundle } \\
& \text { on } \mathcal{O} \text { with flat connection }
\end{array}\right\} .
$$

Given $\gamma=(\gamma, \mathcal{O}) \in \mathscr{D}_{H}$, we have a good notion of locally constant section, thus allowing us to identify $\gamma$ with the sheaf of locally constant sections of $\gamma$. Each $\gamma \in \mathscr{D}_{H}$ has a "size," given by its length

$$
l(\gamma)=\operatorname{dimension} \mathcal{O} .
$$

The following result is well known (cf. [1, 24, 32]).

(1.1) Proposition. The set of irreducible representations in $\mathscr{H}_{F}$ (resp. $\mathcal{O}_{F}^{\prime}$ ) is in one-to-one correspondence with the set $\mathscr{D}_{K}\left(\right.$ resp. $\left.\mathscr{D}_{N}\right)$.

Given $\gamma \in \mathscr{D}_{K}$, we may associate a standard induced from discrete series representation $\pi(\gamma)$ and its unique irreducible quotient $\bar{\pi}(\gamma)$ [32]. The set $\mathscr{D}_{N}$ may be identified with the Weyl group $W$ of $(G, B)$, and in this sense each $w \in \mathscr{D}_{N}$ may be associated with a Verma module $M_{w}$ and its unique irreducible quotient $L_{w}$. Here we follow the conventions of [23]; in particular, $M_{e}=L_{e}$ and $M_{w_{0}}$ contains $F$ as a quotient, where $w_{0}=$ long element of $W$.

\footnotetext{
${ }^{1} \mathrm{~A}$ variation of the conjecture is proved in [38]; see "note added in proof".
} 
Referring to $\$ 2$ for appropriate definitions, we define a geometric Jacquet functor to be an exact covariant functor $J_{\text {geo }}: \mathscr{H}_{\mathscr{C}_{F}} \rightarrow \mathcal{O}_{F}^{\prime}$, which induces a $\mathbf{Z}\left[q^{1 / 2}, q^{-1 / 2}\right]$ module map between the Hecke modules,

$$
\mathbf{J}_{\mathrm{geo}}: \mathscr{M}_{K} \rightarrow \mathscr{M}_{N}
$$

commuting with the action of the Hecke algebra $\mathscr{H}$. Each $\bar{\pi}(\gamma), \gamma \in \mathscr{D}_{K}$, will correspond to a "self-dual" element $\hat{C}_{\gamma} \in \mathscr{M}_{K}$. (Roughly speaking, $\hat{C}_{\gamma}$ is given by the Kazhdan-Lusztig character formula for $\bar{\pi}(\gamma)$, without appropriate \pm 1 's inserted, see §2.) We ask additionally that certain weight filtration data attached to $\mathbf{J}_{\text {geo }}\left(\hat{C}_{\gamma}\right)$ corresponds to a $\mathrm{g}$-module filtration of $J_{\text {geo }}(\bar{\pi}(\gamma))$ with semisimple subquotients. (This is slightly imprecise, but suitable for introductory purposes. See $\S 2$ for the complete formulation.) If we set $q=1$, then for $H=K$ or $N, \mathscr{M}_{H}$ becomes the Grothendieck group $K\left((\cdots)_{F}\right)$ and $\mathbf{J}_{\text {geo }}$ becomes a map of characters which commutes with the coherent continuation action of $W$. In this sense, $\mathbf{J}_{\text {geo }}$ is a " $q$-deformation" of the usual Jacquet functor (2.9). Our first main result is

(1.2) Theorem. If $\gamma \in \mathscr{D}_{K}$, then $\mathbf{J}_{\text {geo }}\left(\hat{C}_{\gamma}\right)$ is computable as an element of $\mathscr{M}_{N}$, via an algorithm. The computation is independent of the geometric Jacquet functor chosen. Moreover, in the sense of Osborne's formula, we have $\Theta(\bar{\pi}(\gamma))=\Theta(J(\bar{\pi}(\gamma)))=$ $\Theta\left(J_{\text {geo }}(\bar{\pi}(\gamma))\right)$.

The proof of (1.2) depends entirely upon the combinatorial considerations of Lusztig-Vogan [24] and Vogan [32], concerning the Kazhdan-Lusztig conjectures. Defining $q^{i / 2}(i \in \mathbf{Z})$ to be weights, (1.2) is taken to say that the weight filtration data of $\mathbf{J}_{\text {geo }}\left(\hat{C}_{\gamma}\right)$ is computable (which by definition corresponds to a $\mathfrak{g}$-filtration with semisimple subquotients of $\left.J_{\text {geo }}(\bar{\pi}(\gamma))\right)$. This "weight terminology" is motivated by our discussion below, concerning Gabber's filtration of mixed perverse sheaves by "weights of Frobenius." It is only fair to remark that (1.2) heavily depends upon deep results from geometry; specifically, Gabber's generalization of Deligne's proof of the Weil conjectures [24].

Up to this point, geometry has not entered our discussion in a direct way. However, to establish the existence of a geometric Jacquet functor, our viewpoint must change. The main result of $\S 3$ is

\section{(1.3) THEOREM. There exists a geometric Jacquet functor $J_{\mathrm{geo}}$.}

The proof of (1.3) is accomplished using the philosophy of "going to positive characteristic," and we will now proceed to sketch the relevant points. The key idea is to introduce the notion of a mixed Jacquet functor $J_{\text {mix }}$. Such a functor is defined between $q$-analogues $\mathscr{H} \mathscr{C}_{q \text {, mix }}$ and $\mathcal{O}_{q \text {, mix }}^{\prime}$, of $\mathscr{H}_{\mathscr{C}_{F}}$ and $\mathcal{O}_{F}^{\prime}$, respectively. Here, $q$ will be almost any prime power and the objects in $\mathscr{H} \mathscr{C}_{q \text {, mix }}$ and $\mathcal{O}_{q \text {, mix }}^{\prime}$ are certain

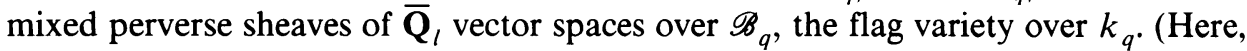
$k_{q}$ is an algebraic closure of the finite field $\mathbf{F}_{q}$.) By going to positive characteristic, the action of the Frobenius map may be encapsulated in the notion of a "mixed category" and we have Gabber's filtration of any such mixed perverse sheaf $F^{*}$, by 
weights of Frobenius, corresponding to a filtration whose subquotients are semisimple and "pure of some weight $i$." The upshot is a diagram of the sort shown in Figure 1.

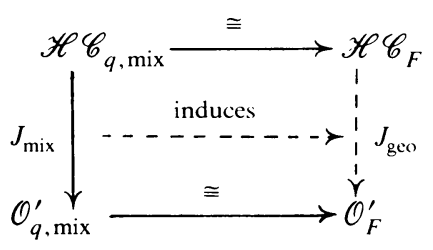

(almost all prime powers $q$ )

\section{Figure 1}

Here, the horizontal equivalences of categories proceed through the RiemannHilbert correspondence and the Beilinson-Bernstein localization theory. We may then induce a functor $J_{\text {geo }}: \mathscr{H}_{\mathscr{C}_{F}} \rightarrow \mathcal{O}_{F}^{\prime}$ which fairly easily has most of the required properties of $J_{\text {geo }}$.

At this point, it is useful to recall that a crucial property of the usual Jacquet functor $J$ is the fact that it commutes with the operation of tensoring by finitedimensional modules [18]. For this reason, the definition of the functor $J_{\text {mix }}$ is modeled on that of $J_{\text {geo }}$, except that the property of commuting with the Hecke algebra action is replaced by a requirement that $J_{\text {mix }}$ commute with the "geometric analogue of $U_{\alpha}$," denoted $\tilde{U}_{\alpha}$. (Since we work in categories of fixed infinitesimal character, this is the appropriate analogue to tensoring by finite-dimensional modules, hence the "right" generalization of $J$.) Extending $J_{\text {mix }}$ to the Grothendieck groups

$$
K\left(\mathscr{H} \mathscr{C}_{q, \text { mix }}\right) \supseteq \mathscr{M}_{K} \quad \text { and } \quad K\left(\mathcal{O}_{q, \text { mix }}^{\prime}\right) \supseteq \mathscr{M}_{N}
$$

for almost all $\dot{q}$, we will be able to induce a $\mathbf{Z}\left[q^{1 / 2}, q^{-1 / 2}\right]$-linear map $\mathbf{J}_{\text {geo }}$ : $\mathscr{M}_{K} \rightarrow \mathscr{M}_{N}$. The work in Lusztig-Vogan [24] tells us that the commutation of $J_{\text {mix }}$ with $\tilde{U}_{\alpha}$ must force $\mathbf{J}_{\text {geo }}$ to be a Hecke algebra map.

In effect, this all shows that (1.3) will follow from the existence of a mixed Jacquet functor $J_{\text {mix }}$. This is established using the local geometry of the Schubert cells in $\mathscr{B}$ and the "vanishing cycles construction" of [2].

At this point, we have the existence and "uniqueness" of a geometric Jacquet functor. Furthermore, $J_{\text {geo }}$ encapsulates all the salient properties of $J$, plus $J_{\text {geo }}$ carries a natural $\mathrm{g}$-filtration with semisimple subquotients. This naturally leads one to the following conjecture; see "note added in proof."

(1.4) Conjecture. The weight filtration data of $\mathbf{J}_{\mathrm{geo}}\left(\hat{C}_{\gamma}\right)$ corresponds to a $\mathfrak{g}$-filtration of $J(\bar{\pi}(\gamma))$ with semisimple subquotients.

Before remarking on the validity of (1.4), it is worthwhile to motivate the importance of such a result. To this end, recall that [18]

$$
H_{*}(\overline{\mathfrak{n}}, X) \cong H_{*}(\overline{\mathrm{n}}, J(X))
$$


$X \in \mathscr{H} \mathscr{C}_{F}$ and $\overline{\mathfrak{n}}$ corresponding to $\theta(N)$. The explicit computation of higher $\overline{\mathrm{n}}$-homology for Harish-Chandra modules is unknown, and (1.5) leads to a category $\mathcal{O}^{\prime}$ approach to the problem. Assuming (1.4) is valid, we immediately glean new information on $H_{0}(\overline{\mathrm{n}}, \bar{\pi}(\gamma)), \gamma \in \mathscr{D}_{K}$. Specifically, any $L_{y}$ in the socle of $J(\bar{\pi}(\gamma))$ will contribute to $H_{0}(\overline{\mathfrak{n}}, \bar{\pi}(\gamma))$; this uses the so-called "self-duality" property of [18]. By Frobenius reciprocity [5], this leads to new information on the structure of principal series representations. Recall that certain pieces of $H_{0}(\overline{\mathrm{n}}, \pi(\gamma))$ - the leading exponents - are known. The exponents described above are the opposite of leading, i.e., they are buried exponents. The leading exponents are typically attached to the middle level of $J(\bar{\pi}(\gamma))$ in (1.4). (These ideas are further developed in [39].)

As a second application, recall [35] that any $L_{y}$ in the socle of $J(\bar{\pi}(\gamma))$ will define the same primitive ideal, i.e., Ann $L_{y}=\operatorname{Ann} \bar{\pi}(\gamma)$. Assuming (1.4), we are led to an algorithm for computing the primitive ideals attached to Harish-Chandra modules. These ideas may be useful in the problem of describing the primitive ideals in some combinatorially uncluttered form.

Our efforts toward (1.4) are less than complete, but sufficient to suggest its validity. \$4 will establish (a strengthened form of) the conjecture in the case of $\mathbf{R}$-rank one and we remark on two R-rank two examples, each of which illustrates the rather delicate nature of our problem. Our methods are very technical, involving case-by-case analysis.

Finally, $\$ 5$ contains an application of our results to the computation of higher $\overline{\mathrm{n}}$-homology. Our example offers a dramatic illustration of the massive cancellation inherent in the Osborne character formula.

In closing, we should point out that many of the results and constructions in this paper were motivated by the situation in $\mathrm{SL}_{2} \mathbf{R}$. For this reason, the reader is strongly urged to frequently consult the examples (2.11) and (3.34).

ACKNOWLEDGMENTS. We are both grateful to H. Hecht and D. Vogan for stirring our initial interest in the structure of Jacquet modules. We also wish to thank P. Deligne for useful discussions on the results of [2]. This work was carried out during the first author's stay at The Institute for Advanced Study, and he wishes to acknowledge gratefully their support. We are also grateful to the referee for his detailed comments on the manuscript.

2. Geometric Jacquet functors: combinatorial aspects. We recall all the notation of the Introduction: $G_{\mathbf{R}}, G, K_{\mathbf{R}}, K, B=H_{m} N$, etc. Additionally, $P_{m, \mathbf{R}}=$ $M_{m, \mathbf{R}} A_{m, \mathbf{R}} N_{m, \mathbf{R}}$ is a compatible minimal parabolic subgroup of $G_{\mathbf{R}}$, i.e., $B \subseteq P_{m}$. Write $G_{\mathbf{R}}^{0}$ for the identity component of $G_{\mathbf{R}}, \mathfrak{g}_{0}=\operatorname{Lie}\left(G_{\mathbf{R}}^{0}\right)$, and $g$ for its complexification; similar notation is used throughout.

Within the category of finite length $\mathfrak{U}(\mathfrak{g})$-modules, we will be interested in two subcategories. By a Harish-Chandra module for $G_{\mathrm{R}}$, we shall mean a module $V$ over the universal enveloping algebra $\mathfrak{U}(\mathfrak{g})$, together with an action of $K_{\mathbf{R}}$, such that: (a) the representation of $K_{\mathbf{R}}$ is an algebraic direct sum of irreducible finite-dimensional continuous representations, each isomorphism class occurring with finite multiplicity; (b) the representation of $\mathfrak{f}$ as a subalgebra of $\mathfrak{g}$ coincides with the complexified 
differential of the $K_{\mathbf{R}}$ action; (c) for any $X \in \mathfrak{H}(\mathfrak{g}), k \in K_{\mathbf{R}}$, and $v \in V$, Ad $k \cdot X$. $v=k \cdot X \cdot k^{-1} \cdot v$; and (d) $V$ has finite length as a $\mathfrak{U}(\mathrm{g})$-module. We put

$$
\mathscr{H} \mathscr{C}=\text { category of Harish-Chandra modules, }
$$

$$
\mathcal{O}^{\prime}=\text { the category of finitely generated } \mathfrak{U}(\mathrm{g}) \text {-modules }
$$
$V$, which are b-finite; i.e., every $v \in V$ lies in a finite-dimensional $\mathfrak{b}$-invariant subspace.

(2.3) Remarks. (i) Any finite-dimensional $K_{\mathbf{R}}$ module extends to a module for $K$; in this sense, Harish-Chandra modules are $(\mathfrak{g}, K)$ modules.

(ii) The category $\mathcal{O}^{\prime}$ is in analogy with the Bernstein-Gelfand-Gelfand category $\mathcal{O}$, but slightly "larger." The point is that we do not demand $\mathfrak{h}_{m}$ acts semisimply. Both categories $\mathcal{O}$ and $\mathcal{O}^{\prime}$ contain the Verma modules and their unique irreducible quotients; the latter forming a basis for both Grothendieck groups $K(\mathcal{O})$ and $K\left(\mathcal{O}^{\prime}\right)$. The category $\mathcal{O}^{\prime}$ carries a natural duality functor, denoted $(\cdots)^{T}$, as defined in [11 or 18]. This is an exact contravariant functor, and $L^{T} \cong L$ for any irreducible $L \in \mathcal{O}^{\prime}$. If $Y \in \mathcal{O}^{\prime}$ has the property that $Y^{T} \cong Y$, we call $Y$ self-dual.

We denote by $\Phi$ the set of roots of $\mathfrak{h}_{m}$ in $g$, and we denote by $\Phi^{+}$the positive root system determined by $B$. Let $W$ denote the Weyl group of $\Phi, l(\cdots)$ the length function on $W$ (relative to $\Phi^{+}$), and let $S$ denote the simple root reflections; we will often identify $S$ with the simple $\Phi^{+}$roots, in the obvious manner. From the Introduction, recall our categories $\mathscr{H} \mathscr{C}_{F}$ and $\mathcal{O}_{F}^{\prime}$.

The Hecke modules. Various "Hecke terminology" is central to all that will follow. Consequently, we give fairly complete definitions. To begin with, consider the Coxeter pair $(W, S)$ and recall that $W$ is generated by $S$, subject to the relations:

$$
\begin{gathered}
s^{2}=1, \quad s \in S, \\
\underbrace{s s^{\prime} s s^{\prime} \cdots s s^{\prime}}_{n\left(s, s^{\prime}\right) \text { factors }}=\underbrace{s^{\prime} s s^{\prime} s \cdots s^{\prime} s,}_{n\left(s^{\prime}, s\right) \text { factors }}, \quad s, s^{\prime} \in S ;
\end{gathered}
$$

here, $n\left(s, s^{\prime}\right)$ is a symmetric function on $S \times S$ taking value 1 (if $s=s^{\prime}$ ), 2 (if $s$ and $s^{\prime}$ commute), 3, 4, or 6 . The Hecke algebra $\mathscr{H}$ of $W$ is the free $\mathbf{Z}\left[q^{1 / 2}, q^{-1 / 2}\right]$ algebra with unit, generated by the elements $\left\{T_{s}: s \in S\right\}$, subject to the relations:

$$
\begin{gathered}
\left(T_{s}+1\right)\left(T_{s}-q\right)=0, \quad s \in S, \\
\underbrace{T_{s} T_{s^{\prime}} T_{s} T_{s^{\prime}} \cdots T_{s} T_{s^{\prime}}}_{n\left(s, s^{\prime}\right) \text { factors }}=\underbrace{T_{s^{\prime}} T_{s} T_{s^{\prime}} T_{s} \cdots T_{s^{\prime}} T_{s}}_{n\left(s^{\prime}, s\right) \text { factors }}, \quad s, s^{\prime} \in S .
\end{gathered}
$$

If $w \in W$, choose a reduced expression $w=s_{1} s_{2} \cdots s_{r}, s_{i} \in S$. Then the element $T_{w}=T_{s_{1}} T_{s_{2}} \cdots T_{s_{r}} \in \mathscr{H}$ is independent of the reduced expression. Moreover, $\mathscr{H}$ is a free $\mathbf{Z}\left[q^{1 / 2}, q^{-1 / 2}\right]$-module with basis $\left\{T_{w}: w \in W\right\}$.

Let $H=K$ or $N$ and recall the sets $\mathscr{D}_{H}$ of the Introduction; these sets parametrize a basis for $K\left(\mathscr{H} \mathscr{C}_{F}\right)$ and $K\left(\mathcal{O}_{F}^{\prime}\right)$. Let $\mathscr{M}_{H}$ be the free $\mathbf{Z}\left[q^{1 / 2}, q^{-1 / 2}\right]$-module with basis $\mathscr{D}_{H}$. We refer to $\mathscr{M}_{H}$ as the Hecke module attached to $\mathscr{D}_{H}$.

(2.4) Lemma. Using [24 and 32], it is possible to define an action of $\mathscr{H}$ on $\mathscr{M}_{K}$ and $\mathscr{M}_{N}$. 
(2.5) Remarks. The exact formulas used to define the Hecke algebra action in (2.4) are somewhat tedious to write out explicitly. To define the action of $T_{s}$ on $\mathscr{M}_{K}$, we must consider the "type of simple root $s$," in the sense of its being compact, real, complex, etc. It is best to think of these formulas as $q$-deformations of the coherent continuation action of $W$ on the standard modules $[32,33]$. Roughly speaking, $T_{s}+1$ corresponds to the $U_{\alpha}$-construction of [30-34], upon setting $q=1$.

The next theorem is at the heart of the proof of the Kazhdan-Lusztig conjectures. Again, $H=K$ or $N$. The set $\mathscr{D}_{H}$ carries an order relation $\leqslant$, the Bruhat G-order; we refer the reader to [24 or 32] for a definition. In the case of $H=N, \leqslant$ is just the Bruhat order.

(2.6) Theorem (Lusztig - Vogan [24, 32]). There exists a unique Z-linear map D: $\mathscr{M}_{H} \rightarrow \mathscr{M}_{H}$, subject to the following conditions:

(a) $D(q m)=q^{-1} D(m), m \in \mathscr{M}_{H}$,

(b) $D\left(\left(T_{s}+1\right) m\right)=q^{-1}\left(T_{s}+1\right) D(m), m \in \mathscr{M}_{H}, s \in S$,

(c) if $\delta \in \mathscr{D}_{H}$, then $D(\delta)=q^{-l(\delta)}\left[\delta+\sum_{\gamma<\delta} R_{\gamma, \delta}(q) \gamma\right]$, where $R_{\gamma, \delta}$ are computable polynomials in $q$ of degree at most $l(\delta)-l(\gamma)$.

Moreover, given $\delta \in \mathscr{D}_{H}$, there exists a unique element

$$
C_{\delta}=\sum_{\gamma \leqslant \delta} P_{\gamma, \delta}(q) \gamma \in \mathscr{M}_{H},
$$

subject to the conditions

(d) $D\left(C_{\delta}\right)=q^{-l(\delta)} C_{\delta}$,

(e) $P_{\delta, \delta} \equiv 1$,

(f) if $\gamma \neq \delta$, then $P_{\gamma, \delta}$ is a computable polynomial in $q$ of degree at most $(l(\delta)-l(\gamma)-1) / 2$.

(2.7) Remarks. (i) We refer to the map $D$ of (2.6) as duality. Loosely speaking, the first part of (2.6) tells us how duality acts on the standard representations, while the second part concerns the action of $D$ on the "irreducibles."

(ii) The elements $C_{\delta}$ of (2.6) are best thought of as the irreducible characters. This is not quite true, since the sum defining $C_{\delta}$ would need to involve $(-1)^{l(\delta)-l(\gamma)}$. Condition (d) in (2.6) tells us that $C_{\delta}$ is "almost" a self-dual basis for $\mathscr{M}_{H}$. Indeed, we set

$$
\hat{C}_{\delta}=q^{-l(\delta) / 2} C_{\delta} \in \mathscr{M}_{H} .
$$

Then $\left\{\hat{C}_{\delta}: \delta \in \mathscr{D}_{H}\right\}$ forms a self-dual basis for $\mathscr{M}_{H}$. As noted in the Introduction, if $H=N$, then we identify $\mathscr{D}_{H}$ with $W$ and hence are led to $C_{w}$ and $\hat{C}_{w}$.

We adopt the viewpoint that $\mathscr{M}_{N}$ is a $q$-deformation of $K\left(\mathcal{O}_{F}^{\prime}\right)$. This leads to a notion of "weighted characters," as follows: Let $X \in \mathscr{M}_{N}$ and write

$$
X=\sum_{w} p_{w}(q) \hat{C}_{w} \quad \text { (finite sum), }
$$

with $p_{w}(q) \in \mathbf{Z}\left[q^{1 / 2}, q^{-1 / 2}\right]$. Regroup coefficients to find $N \in \mathbf{N}$, so that

$$
X=\sum_{n=-N}^{N} q^{n / 2} \underbrace{\left(\sum_{n(i)} a_{w_{n(i)}} \hat{C}_{w_{n(i)}}\right)}_{X_{n}},
$$


with $a_{w_{n(1)}} \in \mathbf{Z}$. We refer to $X_{n}$ as the level of weight $n / 2$. The weight filtration data of $X$ is the data:

$$
\begin{array}{ll}
\text { levels: } & X_{-N}<X_{-N+1}<\cdots<X_{N-1}<X_{N}, \\
\text { weights: } & -N / 2<-N+1 / 2<\cdots<N-1 / 2<N / 2 .
\end{array}
$$

Alternatively, we may represent this by

\begin{tabular}{|c|c}
\hline$X_{N}$ & $N / 2$ \\
$X_{N-1}$ & $N-1 / 2$ \\
\cline { 1 - 1 } & $\vdots$ \\
& $-N+1 / 2$ \\
& $-N / 2$ \\
\cline { 1 - 1 }$X_{-N+1}$ &
\end{tabular}

We encourage the reader to think of (2.8) as a $q$-deformation of the notion of "socle filtration" for objects in category $\mathcal{O}_{F}^{\prime}$.

Geometric Jacquet functors. We are now ready to define the central objects of study in this paper. To orient the reader, it is fruitful to recall the (classical) Jacquet functor $J: \mathscr{H}_{\mathscr{C}_{F}} \rightarrow \mathcal{O}_{F}^{\prime}$,

$$
J(X) \stackrel{\text { def }}{=}\left(\lim \left(X / \overline{\mathfrak{n}}^{k} \cdot X\right)\right)_{\mathfrak{a}_{m} \text {-finite }} \cdot
$$

We now review five important properties of $J$.

Property \#1. $J$ is an exact covariant functor from $\mathscr{H}_{\mathscr{C}_{F}}$ into $\mathcal{O}_{F}^{\prime}$. For a discussion of this result, see [5, 18, 28, or 37].

PROPERTY \#2. J commutes with the coherent continuation action. Recalling the $\varphi_{\alpha}, \psi_{\alpha}$ functors of [31], we formally have

$$
\begin{aligned}
J\left(s_{\alpha} \cdot X\right) & =J\left(\varphi_{\alpha} \psi_{\alpha} X-X\right)=J\left(\varphi_{\alpha} \psi_{\alpha} X\right)-J(X) \\
& =\varphi_{\alpha} \psi_{\alpha} J(X)-J(X)=s_{\alpha} \cdot J(X), \quad X \in \mathscr{H} \mathscr{C}_{F} .
\end{aligned}
$$

The crucial fact is that $J$ commutes with tensoring by finite-dimensional modules $[18,37]$.

PROPERTY \#3. J commutes with the duality $(\cdots)^{T}$ of $(2.3)(\mathrm{ii})$. This result is established in [18]. Its proof strongly depends upon the fact that $J(X)$ may be explicitly constructed from the asymptotic expansions of matrix coefficients (cf. [18] for the definition of $(\cdots)^{T}$ on $\left.\mathscr{H} \mathscr{C}_{F}\right)$.

PROPERTY \#4. $J$ satisfies a support condition. More precisely, if $J(\bar{\pi}(\gamma))=L_{n_{1}}$ $+\cdots+L_{w_{n}}$ in $K\left(\mathcal{O}_{F}^{\prime}\right)$, then $l\left(w_{i}\right) \leqslant l(\gamma), 1 \leqslant i \leqslant n$. This may be seen from Property \#2 and the fact that $\Theta(\bar{\pi}(\gamma))=\Theta(J(\bar{\pi}(\gamma)))$, on some appropriate set (as follows from the Osborne conjecture [17]). This kind of condition was weakly exploited by Schmid in [28].

Property \# 5. $J(\cdots)$ contains the finite-dimensional module $F$ (in $K\left(\mathcal{O}^{\prime}\right)$ ) exactly once in the largest growth situation. This is an easy consequence of the proof of Langlands' classification and Hecht's definition of largest growth representations in [16]. 
Our definition of a geometric Jacquet functor aims to q-deform Properties \# 1\# 5. Precisely,

(2.10) Definition. A geometric Jacquet functor $J_{\text {geo }}$ for $G_{\mathbf{R}}$ is a functor $J_{\text {geo }}: \mathscr{H}_{\mathscr{C}_{F}} \rightarrow \mathcal{O}_{F}^{\prime}$, satisfying the following five axioms:

(G1) (Exactness axiom). $J_{\text {geo }}$ is an exact covariant functor.

(G2) (Coherence axiom). $J_{\text {geo }}$ induces a $\mathbf{Z}\left[q^{1 / 2}, q^{-1 / 2}\right]$-linear map, denoted

$$
\mathbf{J}_{\text {geo }}: \mathscr{M}_{K} \rightarrow \mathscr{M}_{N}
$$

which commutes with the action of the Hecke algebra $\mathscr{H}$. Moreover, to each $\gamma \in \mathscr{D}_{K}, J_{\text {geo }}(\bar{\pi}(\gamma))$ is endowed with a g-module filtration $E_{1} \subset E_{2} \subset \cdots \subset E_{M}$ with semisimple subquotients $E_{i+1} / E_{i}$, corresponding to the respective levels of the weight filtration data for $\mathbf{J}_{\mathrm{geo}}\left(\hat{C}_{\gamma}\right)$.

(G3) (Duality axiom). $\mathbf{J}_{\text {geo }}$ commutes with $D$.

(G4) (Support condition). Let $\gamma \in \mathscr{D}_{K}$ and assume $\hat{C}_{w}, w \in \mathscr{D}_{N}$, occurs in $\mathbf{J}_{\text {geo }}\left(\hat{C}_{\gamma}\right)$. Then $l(w) \leqslant l(\gamma)$.

(G5) (Largest growth axiom). Let $\gamma \in \mathscr{D}_{K}$ be attached to the open $K$-orbit in the flag variety $\mathscr{B}$. Then the finite-dimensional module $F$ occurs exactly once in the weight filtration data for $\mathbf{J}_{\text {geo }}\left(\hat{C}_{\gamma}\right)$. Moreover, it occurs precisely in the zero weight level: $\mathbf{J}_{\text {geo }}\left(\hat{C}_{\gamma}\right)=\hat{C}_{w_{0}}+\sum_{w<w_{0}} p_{w}(q) \hat{C}_{w}$.

(2.11) AN EXAMPLE. To fully orient the reader, we now describe in full detail the case of $G_{\mathbf{R}}=\mathrm{SL}_{2} \mathbf{R}$. In this case, $\mathscr{B}=\mathbf{P}^{1}=\mathbf{C} \cup\{\infty\}$. Identify $\mathrm{SL}_{2} \mathbf{R}=\mathrm{SU}(1,1)$; then $K$ consists of diagonal matrices $\left(\begin{array}{cc}z & 0 \\ 0 & z^{-1}\end{array}\right), z \in \mathbf{C}$. The $K$-action on $\mathscr{B}$ is given by

$$
\left(\begin{array}{ll}
z & 0 \\
0 & z^{-1}
\end{array}\right) \cdot y=z^{2} y, \quad z \in \mathbf{C}, y \in \mathbf{C} \cup\{\infty\} \text {. }
$$

Thus, $\mathscr{B}$ has three $K$-orbits: $\{0\},\{\infty\}$, and $\mathbf{C}^{\times}=$the open $K$-orbit. The isotropy groups $K_{x}$ for $x$ in one of the three orbits are $K, K$, and $\{ \pm I\}$, respectively. The set $\mathscr{D}_{K}$ contains four elements: the constant sheaves on the orbits and a "mobius band" coming from the double cover of $\mathbf{C}^{\times}$. From (1.1), each of these elements may be identified with a standard representation: $\delta_{+}=$principal series which has $F$ as a quotient (corresponds to the trivial sheaf on $\mathbf{C}^{\times}$); $\delta_{-}=$irreducible principal series (corresponds to the "mobius band" on the open orbit); $\delta_{h}=$ a holomorphic discrete series (corresponds to the constant sheaf on $\{0\}$ ); and $\delta_{a}=$ an antiholomorphic discrete series (corresponds to the constant sheaf on $\{\infty\}$ ). Notice that $l\left(\delta_{+}\right)=$ $l\left(\delta_{-}\right)=1$ and $l\left(\delta_{h}\right)=l\left(\delta_{a}\right)=0$.

In a similar way, one can identify $\mathscr{D}_{N}$ with a two-element set: $\delta_{s}=$ Verma module with $F$ as a quotient (corresponds to the open $N$-orbit in $\mathscr{B}$ ) and $\delta_{e}=$ an irreducible Verma module (corresponds to the closed $N$-orbit). Furthermore, we have $l\left(\delta_{s}\right)=1$ and $l\left(\delta_{e}\right)=0$.

We now assume that a geometric Jacquet functor $J_{\text {geo }}$ exists for $G_{\mathbf{R}}=\mathrm{SL}_{2} \mathbf{R}$ (as will be verified in $\S 3$ ). Let us illustrate how to compute the weight filtration data for $\mathbf{J}_{\text {geo }}\left(\hat{C}_{\delta}\right)$. Begin by setting

$$
\mathbf{J}_{\mathrm{geo}}\left(C_{\boldsymbol{\delta}_{-}}\right)=a C_{s}+b C_{e},
$$


where $a, b \in \mathbf{Z}\left[q^{1 / 2}, q^{-1 / 2}\right]$; we are identifying $s \leftrightarrow \delta_{s}, e \leftrightarrow \delta_{e}$, and $W=\{e, s\}$. From [23, 24, and 32],

$$
\begin{aligned}
& \text { (a) }\left(T_{s}+1\right) \cdot \delta_{-}=0, \\
& \text { (b) }\left(T_{s}+1\right) \cdot C_{s}=(1+q) C_{s}, \\
& \text { (c) }\left(T_{s}+1\right) \cdot C_{e}=C_{s} .
\end{aligned}
$$

Combining (2.12), (2.13), and (2.10)(G2), we find

$$
\begin{aligned}
0 & =\mathbf{J}_{\mathrm{geo}}\left(\left(T_{s}+1\right) \cdot \delta_{-}\right)=\left(T_{s}+1\right) \mathbf{J}_{\mathrm{geo}}\left(C_{\delta}\right) \\
& =\left(T_{s}+1\right)\left(a C_{s}+b C_{e}\right)=(a+a q+b) C_{s} .
\end{aligned}
$$

This forces $b=-(1+q) a$ and so

$$
\mathbf{J}_{\text {geo }}\left(C_{\delta_{-}}\right)=a\left(C_{s}-(1+q) C_{e}\right) .
$$

Since $\delta_{-}$is a largest growth representation [16], (2.10)(G5) ensures that $a \equiv 1$. Finally,

$$
\begin{aligned}
\mathbf{J}_{\mathrm{geo}}\left(\hat{C}_{\delta_{-}}\right) & =\mathbf{J}_{\mathrm{geo}}\left(q^{-1 / 2} C_{\delta}\right)=q^{-1 / 2} \mathbf{J}_{\mathrm{geo}}\left(C_{\delta_{-}}\right) \\
& =q^{-1 / 2} C_{s}-q^{-1 / 2} C_{e}-q^{1 / 2} C_{e} \\
& =-q^{-1 / 2} \hat{C}_{e}+q^{0} \hat{C}_{s}-q^{1 / 2} \hat{C}_{e} .
\end{aligned}
$$

This gives weight filtration data:

$$
\mathbf{J}_{\mathrm{geo}}\left(\hat{C}_{\delta_{-}}\right)=\begin{array}{|l|l}
\hline-\hat{C}_{e} & 1 / 2 \\
\cline { 1 - 1 } & 0 \\
\cline { 1 - 1 } \hat{C}_{s} & 0 \\
\cline { 1 - 1 } \hat{C}_{e} & -1 / 2 \\
\hline
\end{array}
$$

The observation to be made is that (2.14) will correspond to the socle filtration of $J\left(\bar{\pi}\left(\delta_{-}\right)\right)$. Precisely, as is described in [18], we have

$$
J\left(\bar{\pi}\left(\delta_{-}\right)\right)=\begin{array}{|l|}
\hline L_{e} \\
\hline L_{s} \\
\hline L_{e} \\
\hline
\end{array},
$$

where $L_{e}=\operatorname{socle} J\left(\bar{\pi}\left(\delta_{-}\right)\right), L_{s}=\operatorname{socle}\left(J\left(\bar{\pi}\left(\delta_{-}\right)\right) / L_{e}\right), L_{e}=\operatorname{top} J\left(\bar{\pi}\left(\delta_{-}\right)\right)$. The correspondence can be made even more precise by inserting the appropriate \pm 1 's in $\hat{C}_{\delta_{-}}$. This "match up" was our original motivation for studying geometric Jacquet functors. For completeness, one can also check that $\mathbf{J}_{\text {geo }}\left(\hat{C}_{\delta_{h}}\right)=\mathbf{J}_{\text {geo }}\left(\hat{C}_{\delta_{a}}\right)=\hat{C}_{e}$ and $J\left(\bar{\pi}\left(\delta_{a}\right)\right)=J\left(\bar{\pi}\left(\delta_{h}\right)\right)=L_{e}$. This verifies conjecture (1.4), in the case of $G_{\mathbf{R}}=\mathrm{SL}_{2} \mathbf{R}$.

The uniqueness theorem. We now come to the first main result of the paper. It will assert that the computation of $\mathbf{J}_{\text {geo }}\left(\hat{C}_{\delta}\right) \in \mathscr{M}_{N}$ is independent of the geometric Jacquet functor chosen.

(2.15) THEOREM. Fix a geometric Jacquet functor $J_{\text {geo }}$ for $G_{\mathbf{R}}, \delta \in \mathscr{D}_{K}$, and $\hat{C}_{\delta} \in \mathscr{M}_{K}$. Then there exists an algorithm to compute the weight filtration data of $\mathbf{J}_{\text {geo }}\left(\hat{C}_{\delta}\right)$ in $\mathscr{M}_{N}$. Moreover, this weight filtration data is independent of the choice of geometric Jacquet functor. 
Note. Keep in mind that we do not yet know any such $J_{\text {geo }}$ exists. This will be verified in $\S 3$. We should also point out that the duality axiom in (2.10) will not enter into the proof of $(2.15)$, though all the others will. That axiom was included mainly to emphasize the analogy with the usual Jacquet functor $J$.

Proof of (2.15). Assume that we can give an algorithm which succeeds to compute the weight filtraiton of $\mathbf{J}_{\text {geo }}(\delta), \delta \in \mathscr{D}_{K}$. Then, because

$$
C_{\delta}=\sum_{\gamma \leqslant \delta} P_{\gamma, \delta}(q) \gamma
$$

with $P_{\gamma, \delta}(q)$ the Kazhdan-Lusztig polynomials for $G_{\mathbf{R}}$ (as in (2.6)), $\mathbf{J}_{\text {geo }}\left(C_{\delta}\right)=$ $\sum_{\gamma \leqslant \delta} P_{\gamma, \delta}(q) \mathbf{J}_{\text {geo }}(\gamma)$ will be computable. Thus, fix $\delta \in \mathscr{D}_{K}$ and write

$$
\mathbf{J}_{\text {geo }}(\delta)=\sum_{w \in \mathscr{D}_{N}} a_{w}(q) C_{w}, \quad a_{w} \in \mathbf{Z}\left[q^{1 / 2}, q^{-1 / 2}\right] .
$$

Our proof is reduced to giving an algorithm for computing the $a_{w}$ 's.

Begin by letting $w_{1}^{i}, i=1,2, \ldots, m_{1}$, be the elements of $W$ of maximal length occurring in the sum (2.16). Fix a particular $w_{1}^{i}$ and let $w=w(i)$ be of minimal length such that: If $w=s_{1} \cdots s_{r}=w_{0}\left(w_{1}^{i}\right)^{-1}$, then

$$
\underbrace{\left(T_{s_{1}}+1\right)\left(T_{s_{2}}+1\right) \cdots\left(T_{s_{r}}+1\right)}_{\begin{array}{c}
\| \text { def } \\
A
\end{array}} \cdot C_{w_{1}^{i}}=a C_{w_{0}}+\text { smaller terms, } \quad a \neq 0,
$$

$a \in \mathbf{Z}\left[q^{1 / 2}, q^{-1 / 2}\right]$. Furthermore, we can (and do) suppose that $l\left(w_{1}^{i}\right)<l\left(s_{r} w_{1}^{i}\right)<$ $\cdots<l\left(w w_{1}^{i}\right)=l\left(w_{0}\right)=$ dimension of the open $N$-orbit in $\mathscr{B}$. Using the formulas in [23], it will follow that $a \equiv 1$ and for any other $y$ occurring in (2.16), $A \cdot C_{y}$ does not involve $C_{w_{0}}$. (If it did, then length considerations would force $y=w_{1}^{j}$, for some $j$. Then $w y=w w_{1}^{i}$ forces $i=j$.) By $(2.10)(\mathrm{G} 2)$,

$$
\mathbf{J}_{\mathrm{geo}}(A \cdot \delta)=A \cdot \mathbf{J}_{\mathrm{geo}}(\delta)=a_{w_{1}^{i}}(q) C_{w_{0}}+\text { smaller terms. }
$$

By $(2.10)(\mathrm{G} 4),(\mathrm{G} 5) w$ will be of minimal length so that $A \cdot \delta$ has some constituent(s) supported on the open orbit. Furthermore, if $s=\operatorname{dim} \mathscr{B}$,

$$
A \cdot \delta=\left(\sum_{l(\gamma)=s} a_{\gamma}(q) \gamma\right)+\text { smaller terms, }
$$

with the first sum (the leading term) being computable from Lusztig-Vogan [24] or Vogan [32]. Combine (2.17), (2.18), and (2.10)(G5) to show

$$
a_{w_{1}^{i}}(q)=\sum_{l(\gamma)=s} a_{\gamma}(q)
$$

The argument above applies to each $w_{1}^{i}$ individually, for $1 \leqslant i \leqslant m_{1}$, leading to the computation of all $a_{w_{1}^{i}}$ 's, as in (2.19).

Inductively, suppose $w_{k}^{i}, 1 \leqslant i \leqslant m_{k}$, are the elements in $W$ occurring in (2.16) of length $l_{1}-(k-1)$; here $l_{1}=l\left(w_{1}^{i}\right), 1 \leqslant i \leqslant m_{1}$. Assume we have computed $a_{w_{n}^{i}}$ for $n<k$, and fix $w_{k}^{j}$. Let $w^{\prime} \in W$ have minimal length so that: If $w^{\prime}=s_{1} \cdots s_{t}$, then

$$
\underbrace{\left(T_{s_{1}}+1\right)\left(T_{s_{2}}+1\right) \cdots\left(T_{s_{t}}+1\right)}_{A^{\prime}} \cdot C_{w_{k}^{j}}=C_{w_{0}}+\text { smaller terms. }
$$


We have that

$$
A^{\prime} \cdot \delta=\left(\sum_{l(\gamma)=s} a_{\gamma}^{\prime}(q) \gamma\right)+\text { smaller terms, }
$$

with $a_{\gamma}^{\prime} \in \mathbf{Z}\left[q^{1 / 2}, q^{-1 / 2}\right]$. Again, [ 24 or 32] ensure the first term of (2.21) is computable. Put

$$
\mathbf{J}_{\text {geo }}(\delta)=\sum_{n<k} a_{w_{n}^{\prime}} C_{w_{n}^{\prime}}+a_{w_{k}^{\prime}} C_{w_{k}^{\prime}}+\sum_{\substack{r \geqslant k \\ w_{k}^{\prime} \neq w_{k}^{\prime}}} a_{w_{r}^{\prime}} C_{w_{r}^{\prime}} .
$$

Let $b(q)=$ coefficient of $C_{k^{\prime} 0}$ in the expression

$$
A^{\prime}\left(\sum_{n<k} a_{w_{n}^{\prime}} C_{k_{n}^{\prime}}\right)=b(q) C_{w_{i,}}+\text { smaller terms. }
$$

Notice that $b(q)$ is computable, since we inductively know all $a_{w_{n}^{i}}, n<k$. Combine (2.21) with $(2.10)(\mathrm{G} 4),(\mathrm{G} 5)$ to find a computable $a(q)$, which satisfies

$$
\mathbf{J}_{\text {geo }}\left(A^{\prime} \cdot \delta\right)=a(q) C_{w_{0}}+\text { smaller terms. }
$$

By the choice of $A^{\prime},(2.10)(\mathrm{G} 2),(2.23)$, and (2.24), we see

$$
\begin{aligned}
A^{\prime} \cdot \mathbf{J}_{\text {geo }}(\delta) & =A^{\prime}\left(\sum_{n<k} a_{w_{n}^{\prime}} C_{w_{n}^{\prime}}\right)+a_{w_{k}^{\prime}} C_{w_{0}}+\text { smaller terms } \\
& =b(q) C_{w_{0}}+a_{w_{k}^{\prime}}(q) C_{w_{0}}+\text { smaller terms } \\
& =a(q) C_{\left.w_{0}\right)}+\text { smaller terms }=\mathbf{J}_{\text {geo }}\left(A^{\prime} \cdot \delta\right) .
\end{aligned}
$$

The upshot of this computation is the formula

$$
a_{w_{h}^{\prime}}(q)=a(q)-b(q),
$$

which is computable. Repeat this argument for all $w_{k}^{i}, 1 \leqslant i \leqslant m_{k}$, to reach a formula as in (2.25), thus computing all $a_{w_{k}^{\prime}}$ 's. By induction, we now have computed the decomposition in (2.16).

Finally, one should note that the properties needed to compute $\mathbf{J}_{\text {geo }}\left(\hat{C}_{\delta}\right) \in \mathscr{M}_{N}$ were axiomatic, so our theorem is proved. Q.E.D.

In closing, it should be noted that this argument tells us something about the relationship between the classical Jacquet functor $J$ and $J_{\text {geo }}$. Precisely,

(2.26) Scholium. We have $\Theta(\bar{\pi}(\delta))=\Theta(J(\bar{\pi}(\delta)))=\Theta\left(J_{\text {geo }}(\bar{\pi}(\delta))\right), \delta \in \mathscr{D}_{K}$, in the sense of Osborne's formula [17].

Proof. If we set $q=1$ in $\mathscr{M}_{H}(H=K, N)$, the Hecke algebra action becomes coherent continuation (cf. [24]) on $K\left((\cdots)_{H}\right)$. The proof of (2.15) then shows the formal characters $\Theta(J(\bar{\pi}(\delta)))$ and $\Theta\left(J_{\text {geo }}(\bar{\pi}(\delta))\right)$ agree as asserted. The final equality is a consequence of Osborne's conjecture (a theorem of Hecht-Schmid [17]); cf. [18 or 6, (4.2)]. Q.E.D.

3. Geometric Jacquet functors: geometric aspects. The objective of this section is to produce an example of a geometric Jacquet functor, having all the essential features of the usual Jacquet functor $J$ (2.9), and realizing the weight filtration data of $\S 2$, as 
a filtration with semisimple subquotients. Our original intention was to give a geometric construction of the usual $J$ (thus proving conjecture (1.4)). This project is not accomplished here. ${ }^{2}$ However, the new object we construct is designed to satisfy the fundamental properties of $J$ with the hope that these characterize the usual functor $J$, in some appropriate sense. Technically, the geometric construction of an analogue of the Jacquet functor proves the existence of the unique map of Hecke algebras $\mathbf{J}_{\text {geo }}$ which was "computed" in $\S 2$. It also shows that $\mathbf{J}_{\text {geo }}$ commutes with $D$, the duality operation. This is because it is induced, in some precise geometric way, by a functor commuting with Verdier duality, thus completing the proof of Theorem (1.3).

Our construction of a geometric analogue of the Jacquet functor makes use of the Beilinson-Bernstein theory, so we have included a brief account of it. Also, the weight filtration theorem of $\mathrm{O}$. Gabber [2, Theorem 5.3.5] will be crucial; this generalizes the well-known Decomposition theorem for $D$-modules.

Beilinson-Bernstein theory. Throughout this section we will have to view an algebraic variety $Y$ over $\mathbf{C}$ as an object endowed with, respectively, three different structures: $Y^{\text {alg }}=Y$ is the algebraic variety of $Y$ with Zariski topology; $Y^{\text {an }}$ consists of the complex points of $Y$ viewed as a complex analytic set, and $Y^{\text {et }}$ is $Y$ with its etale topology [27]. Often we will consider derived categories over $Y^{\mathrm{et}}, Y^{\text {an }}$, consisting of complexes of sheaves with constructible cohomology. If $\pi$ is an algebraic map between two varieties, then $\pi_{*}, \pi_{!}$, etc., will denote the direct image, direct image with proper supports, etc., as in [2] taken in the derived category, instead of using the notation $R \pi_{*}, R \pi_{\text {! }}$, etc.

Recall that we are fixing throughoui this paper a finite-dimensional representation $F$ of $G_{\mathbf{R}}$. Let $\mathscr{L}_{F}$ be a homogeneous line bundle over $\mathscr{B}^{\text {alg }}$, realizing $F$ on its global section (as in the Borel-Weil theorem), $\mathcal{O}_{F}$ the sheaf of sections of $\mathscr{L}_{F}$ and $D_{F}$ the sheaf of twisted differential operators on $\mathcal{O}_{F}$.

We denote by $\mathscr{M}\left(D_{F}\right)$ the category of left $D_{F}$-modules, and by $\mathscr{M}(\mathfrak{g})_{F}$ the category of $g$-modules with the same infinitesimal character as $F$. It should be remarked that when $F$ is trivial, then $D_{F}=D_{\mathscr{B}}$ is the sheaf of differential operators on $\mathscr{B}$ with coefficients in the structure sheaf $\mathcal{O}_{\mathscr{B}}$. The theory of $D$-modules, as it often appears in the literature, applies to modules over $D_{\mathscr{B}}$, rather than over its twisted counterpart $D_{F}$; for this reason we include the following theorem connecting $D_{F}$-modules to $D_{\mathscr{B}}$-modules whose proof is left to the reader [26].

(3.1) THEOREM. There is an equivalence of categories

$$
\mathscr{M}\left(D_{\mathscr{B}}\right) \stackrel{\psi_{F}}{\rightarrow} \mathscr{M}\left(D_{F}\right), \quad \psi_{F}(M)=\mathcal{O}_{F} \otimes_{\mathcal{O}_{\mathscr{P}}} M
$$

Under the equivalence of (3.1), regular singular holonomic $D_{F}$-modules correspond to regular singular holonomic $D_{\mathscr{B}}$-modules in the usual sense. Denote by $\mathscr{M}\left(D_{F}\right)_{\mathrm{RS}}$ the category of regular singular $D_{F}$-modules. From [1] we have the following theorem which is a crucial step in the proof of the Kazhdan-Lusztig conjectures.

\footnotetext{
${ }^{2}$ See "note added in proof" for recent work.
} 
(3.3) THEOREM (BEILINSON - BERNSTEIN). There is an equivalence of categories

$$
\Delta: \mathscr{M}(\mathfrak{g})_{F} \rightarrow \mathscr{M}\left(D_{F}\right), \quad \Delta(M)=D_{F} \otimes_{\mathfrak{l}(\mathfrak{g})} M .
$$

Under $\Delta$, the categories $\mathscr{H}_{\mathscr{C}_{F}}, \mathcal{O}_{F}^{\prime}$ become categories of holonomic $D_{F}$-modules with regular singularities.

Note that the tensor product $D_{F} \otimes_{\mathfrak{u}(g)} M$ makes sense because the enveloping algebra maps into the global sections of $D_{F}$. The categories in the image of $\Delta$ obtained from $\mathscr{H}_{\mathscr{C}_{F}}$ and $\mathcal{O}_{F}^{\prime}$ will be denoted, respectively, $\mathscr{H} \mathscr{C}\left(D_{F}\right)$ and $\mathcal{O}^{\prime}\left(D_{F}\right)$.

Riemann-Hilbert correspondence. From here on, we will be using several versions of the sets $\mathscr{D}_{K}, \mathscr{D}_{N}$ introduced in $\S 1$. A new set $\mathscr{D}_{H}^{\text {new }}, H=K$ or $N$, will always be in some canonical bijective correspondence to $\mathscr{D}_{H}^{\text {old }}$, but for convenience both will simply be denoted by $\mathscr{D}_{H}$. Until we say otherwise,

$$
\mathscr{D}_{H}=\left\{\begin{array}{l}
(\mathcal{O}, \mathscr{L}), \mathcal{O} \text { is an } H \text {-orbit on } \mathscr{B}^{\text {an }}, \\
\mathscr{L} \text { is an } H \text {-equivariant sheaf of } \mathrm{C} \text {-vector } \\
\text { spaces with stalks of dimension one. }
\end{array}\right.
$$

Let $\mathscr{C}$ be the category of sheaves on $\mathscr{B}^{\text {an }}$ which are constructible over an algebraic stratification on $\mathscr{B}^{\text {an }}$ (the strata are algebraic nonsingular varieties). Denote by $D(\mathscr{C})$ the derived category of doubly bounded complexes of sheaves on $\mathscr{B}^{\text {an }}$ with cohomology in $\mathscr{C}$. Inside this category there is an abelian subcategory, the category of perverse sheaves $\operatorname{Perv}(\mathscr{C})$, consisting of complexes $F^{*}$ of sheaves which satisfy the perversity conditions:

(PI) $\mathscr{H}^{i} F^{\cdot}=0$ for $i>0$;

(PII) dim support $\mathscr{H}^{i}\left(F^{\bullet}\right) \leqslant-i$;

(PIII) The Verdier dual of $F^{*}$, denoted $D F^{*}$ satisfies (PI) and (PII).

The objects in $\operatorname{Perv}(\mathscr{C})$ all have finite length and the irreducible objects can be described by very simple conditions $[2$, p. 112]. Let $\mathscr{L}$ be a locally constant sheaf over a nonsingular algebraic subvariety $Y$ of $\mathscr{B}^{\text {an }}$. In [12] Goresky and MacPherson intróduced a complex of sheaves that can be associated to $\mathscr{L}$. This complex, denoted $I C(\mathscr{L})$, lives on all of $\mathscr{B}^{\text {an }}$ and is characterized by the following properties. $F^{*}=I C(\mathscr{L})$ if and only if support $F^{*} \subseteq \bar{Y}$ and

(ICI) $\mathscr{H}^{i} F^{\cdot}=0$ for $i \notin\{i \in \mathbf{Z}: 0 \leqslant-i \leqslant \operatorname{dim} Y\}$;

(ICII) $\operatorname{dim}$ support $\mathscr{H}^{i}\left(F^{*}\right)<-i$ for $0 \leqslant-i<\operatorname{dim} Y$;

(IC III) $\left.F^{\cdot}\right|_{Y}=\mathscr{L}[\operatorname{dim} Y]$

(ICIV) $D F^{*}$, the Verdier dual, satisfies (ICI), (ICII), (IC III).

(3.4) THEOREM [1 AND 2, (4.3.1)]. The irreducible objects in $\operatorname{Perv}(\mathscr{C})$ are precisely the intersection cohomology complexes $I C(\mathscr{L}), \mathscr{L}$ a locally constant sheaf over a nonsingular subvariety $Y$ of $\mathscr{B}^{\text {an }}$.

We introduce some additional notation in preparation for the Riemann-Hilbert correspondence (3.5). We let

$D_{\mathscr{B}}^{\text {an }}=$ The sheaf of differential operators on $\mathscr{B}^{\text {an }}$ with holomorphic coefficients (contains $D_{\mathscr{B}}$ ),

$\Omega_{\mathscr{D}}^{\text {an }}=$ The right $D_{\mathscr{B}}^{\text {an }}$-module consisting of holomorphic forms of the top degree on $\mathscr{B}$. 
If $M$ is a $D_{\mathscr{D}}$-module, the de Rham complex of $M$ is the complex of sheaves on $\mathscr{B}^{\text {an }}$

$$
\operatorname{DR}(M) \stackrel{\text { def }}{=} \Omega_{\mathscr{B}}^{\text {an }} \underset{D_{\mathscr{P}}}{\bigotimes} M
$$

The following is an algebraic version of the Riemann-Hilbert correspondence in [22, 25, and 4, Corollary 4.2.4].

(3.5) THEOREM. There is an equivalence of categories

$$
\mathscr{M}\left(D_{\mathscr{B}}\right)_{\mathrm{RS}} \stackrel{\mathrm{DR}}{\rightarrow} \operatorname{Perv}(\mathscr{C}) .
$$

Putting together the equivalence of categories in (3.1), (3.3), and (3.5), plus (3.4), we find: $\operatorname{Perv}(\mathscr{C})$ corresponds to certain $\mathfrak{g}$-modules of finite length with the same infinitesimal character as $F$, and the complexes $I C(\mathscr{L})$ become irreducible g-modules. In particular, as in [32 and 1], if $\mathscr{L}$ is in $\mathscr{D}_{K}, I C(\mathscr{L})$ corresponds to an irreducible in $\mathscr{H} \mathscr{C}_{F}$; if $\mathscr{L}$ is in $\mathscr{D}_{N}$, then $\operatorname{IC}(\mathscr{L})$ becomes an irreducible in $\mathcal{O}^{\prime}$. These facts are used in the proof of the Kazhdan-Lusztig conjectures in the two categories [32, 1].

The complexes $I C(\mathscr{L})$ carry $H$-actions, with $H=K$ or $N$ in the following sense: Let $H$ be a group acting on a variety $Y$. A perverse sheaf $F^{*}$ on $Y$ has an $H$-action (or is $H$-equivariant) if there is an isomorphism $\alpha$ in the derived category

$$
\alpha: p^{*} F^{\cdot} \rightarrow \operatorname{act}^{*} F^{*}
$$

with $p$ and act the maps $H \times Y \rightarrow Y$ given by: $p(x, y)=y$, act $(x, y)=x \cdot y$. The only condition on $\alpha$ is that if it is restricted to $\{e\} \times Y$ ( $e$ the identity in $H$ ), the new map obtained is the class of the identity from $F^{*}$ to itself. If $F^{*}$ is locally constant, and $Y$ is an $H$-orbit, then $F^{*}$ with its $H$-action is determined by a finite-dimensional representation of the isotropy group of $H$, trivial on the corresponding identity component. We let $\mathscr{H}_{\mathscr{C}_{\text {perv }}}, \mathcal{O}_{\text {perv }}^{\prime}$ be the categories of perverse sheaves on $\mathscr{B}^{\text {an }}$ with a $K$-action (respectively $N$-action). In particular, the restrictions of the complexes in these two categories to the corresponding orbits under $K$ or $N$ have cohomology consisting of successive extensions of sheaves in $\mathscr{D}_{K}$ (or $\mathscr{D}_{N}$ ). As in [1], $\mathscr{H}_{\mathscr{C}_{F}}$ (or $\mathcal{O}_{F}^{\prime}$ ) corresponds to the category of $\left(D_{F}, K\right)$-modules (or $\left(D_{F}, N\right)$-modules) under localization. Hence we obtain

(3.6) COROLlaRY. There are equivalences of categories

$$
\mathscr{H} \mathscr{C}_{F} \approx \mathscr{H} \mathscr{C}_{\text {perv }} \text { and } \mathcal{O}_{F}^{\prime} \approx \mathcal{O}_{\text {perv }}^{\prime} .
$$

Reduction to positive characteristic. Let $\mathbf{F}_{q}$ be the field with $q$ elements, $q=p^{r}$, with $p$ a prime number, and let $k=k_{q}$ be an algebraic closure of $\mathbf{F}_{q}$. If $Y$ is a complex variety defined over a finite extension of $\mathbf{Q}$, by certain sets of polynomials $S_{Y}$ (by equations and inequalities), it is possible to obtain a new variety $Y_{q}$ over a field $k=k_{q}$; this is done by reducing modulo $p$ the coefficients involved in the polynomials of $S_{Y}$. By this procedure one can obtain, starting from $Y$, varieties $Y_{q}$ 
for all but a finite number of characteristics $p$, which have similar properties as $Y$. In particular, from our group $G$, we obtain $G_{q}$ defined over $\mathbf{F}_{q}$ and

(a) each class of parabolic subgroups in $G_{q}$ is defined over $\mathbf{F}_{q}$ (including our fixed group $B_{q}$ ),

(b) $K_{q}$ and $\boldsymbol{\theta}$ (the Cartan involution) are defined over $\mathbf{F}_{q}$,

(c) each $K_{q}$-orbit on $\mathscr{B}_{q}$ is defined over $\mathbf{F}_{q}$,

(d) each $N_{q}$-orbit on $\mathscr{B}_{q}$ is defined over $\mathbf{F}_{q}$.

The Weyl group $W_{q}$ defined with respect to an $\mathbf{F}_{q}$-split torus in $B_{q}$ can be identified to the Weyl group $W$ of $G$, so we just denote it by $W$. We also recall our set $S \subset W$ of simple reflections.

Let $l$ be a prime number, prime to $q$. The sets $\mathscr{D}_{K}, \mathscr{D}_{N}$ now become $H$-equi-

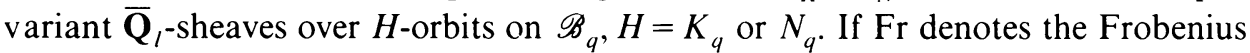
morphism, given coordinate-wise by $\operatorname{Fr}(x)=x^{q}$, Fr gives a homeomorphism in the etale topology of all our varieties defined over $\mathbf{F}_{q}$. We assume that Fr acts trivially on $\mathscr{D}_{K}, \mathscr{D}_{N} ;$ in the sense of [24].

We will now define a $q$-analogue of the categories $\mathscr{H} \mathscr{C}_{\text {perv }}$ and $\mathcal{O}_{\text {perv }}^{\prime}$, referring to [2 and 10] for precise definitions. Let $\mathscr{H}_{\mathscr{C}_{q}}, \mathcal{O}_{q}^{\prime}$ be the categories of $H$-equivariant perverse $\overline{\mathbf{Q}}_{1}$-sheaves on $\mathscr{B}_{q}, H=K_{q}$ or $N_{q}$. Thus we are considering complexes of sheaves of $\overline{\mathbf{Q}}_{l}$-vector spaces in the etale topology on $\mathscr{B}_{q}$, satisfying the perversity conditions (PI), (PII), (PIII), and the $H$-equivariance guarantees that on the $H$-orbits, the cohomology of our complexes consists of sheaves which are successive extensions of elements in $\mathscr{D}_{H}$. By taking into account the action of Fr, we may also consider mixed versions of these categories: Let $\mathscr{H}_{\mathscr{C}_{q, \text { mix }}}, \mathcal{O}_{q, \text { mix }}^{\prime}$ be the abelian categories of

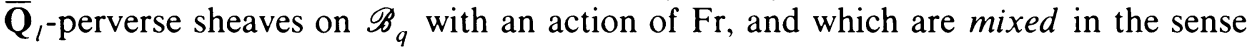
of [10]. The term "mixed" means that the cohomology sheaves over the $H$-orbits are successive extensions of locally constant $\overline{\mathbf{Q}}_{l}$-sheaves, where Fr acts by eigenvalues $\lambda$, with complex absolute value $|\lambda|_{\mathbf{C}} \in\left\{q^{i / 2}: i \in \mathbf{Z}\right\}$ over all the points fixed by $\mathrm{Fr}$. There is a forgetful functor which ignores the Fr actions:

$$
\text { For: } \begin{aligned}
\mathscr{H}_{\mathscr{C}_{q, \text { mix }}} & \rightarrow \mathscr{H} \mathscr{C}_{q} \\
\mathcal{O}_{q, \text { mix }} & \rightarrow \mathscr{O}_{q}^{\prime}
\end{aligned}
$$

From the comparision theorems in $[2, \S 6]$, one obtains

(3.7) THEOREM. There are equivalences of categories

$$
\mathscr{H} \mathscr{C}_{\text {perv }} \approx \mathscr{H} \mathscr{C}_{q} \text { and } \mathcal{O}_{\text {perv }}^{\prime} \approx \mathcal{O}_{q}^{\prime}
$$

for all but a finite number of prime numbers $p$, where $q=p^{r}$.

Gabber's weight filtration. We recall some definitions from [10]. A complex $F^{*}$ of $\overline{\mathbf{Q}}_{l}$-sheaves which is mixed is said to satisfy $\omega\left(F^{*}\right) \leqslant l$ if $\mathscr{H}^{i}\left(F^{*}\right)$ is a sheaf where $\mathrm{Fr}$ acts with eigenvalues $\lambda$ of absolute value $|\lambda|_{\mathbf{C}} \leqslant q^{(i+l) / 2}$ over the points fixed by Fr. It is said to satisfy $\omega\left(F^{*}\right) \geqslant l$ if $\omega\left(D F^{*}\right) \leqslant-l$. And whenever $\omega\left(F^{*}\right) \leqslant l$ and $\omega\left(F^{*}\right) \geqslant l, F^{*}$ is said to be pure of weight $l / 2$. For example, all the irreducibles $I C(\mathscr{L})$ of our categories $\mathscr{H} \mathscr{C}_{q, \text { mix }}, \mathcal{O}_{q \text {, mix }}$ are pure of a certain weight of the form $q^{m / 2}[2,(5.3 .4)]$. The following theorem is a particular case of the weight filtration theorem of O. Gabber [2, (5.3.5)]. 
(3.8) TheOREM. Let $F^{*}$ be an object of $\mathscr{H}_{q, \text { mix }}$ or $\mathscr{O}_{q, \text { mix }}^{\prime}$. Then there is an increasing filtration

$$
0=F_{r} \subset F_{r+1} \subset \cdots \subset F_{m}=F^{*}
$$

which is also increasing in the weights $\omega\left(F_{i}\right) \leqslant i$. The subquotients $F_{i+1} / F_{i}$ are semisimple and pure of weight $(i+1) / 2$ for $i=r, \ldots, m-1$.

A filtration as in (3.8) will be called a weight filtration of $F^{*}$.

Grothendieck groups. Let $\mathscr{C}_{H, q, \text { mix }}^{\prime}, H=K_{q}$ or $N_{q}$, be the categories of constructible $H$-equivariant mixed sheaves on $\mathscr{B}_{q}$. A typical object in these categories consists of a pair $(F, \Phi)$, with $\Phi$ the isomorphism defining the action of $\mathrm{Fr}$,

$$
\operatorname{Fr}^{*}(F) \stackrel{\oplus}{\rightarrow} F,
$$

and $\Phi$ being $H$-equivariant as in [24, (2.2)].

We now obtain new categories $\mathscr{C}_{H, q \text {, mix }}$, by making certain identifications: Two objects $\left(F, \Phi_{1}\right),\left(F, \Phi_{2}\right)$ are equivalent if $\Phi_{1}^{n}=\Phi_{2}^{n}$ for some $n$. The isomorphisms in these categories are maps of $H$-equivariant $l$-adic sheaves, compatible with corresponding $\Phi^{n}$ for some $n$. On the level of Grothendieck groups we have maps

$$
\begin{aligned}
K\left(\mathscr{H} \mathscr{C}_{q, \text { mix }}\right) \underset{\mathbb{1}}{\stackrel{\approx}{\rightarrow}} K\left(\mathscr{C}_{K, q, \text { mix }}^{\prime}\right) \rightarrow K\left(\mathscr{C}_{K, q, \text { mix }}\right), \\
K\left(\mathscr{O}_{q, \text { mix }}^{\prime}\right) \underset{\mathbb{1}}{\stackrel{\sim}{\rightarrow}} K\left(\mathscr{C}_{N, q, \text { mix }}^{\prime}\right) \rightarrow K\left(\mathscr{C}_{N, q, \text { mix }}\right)
\end{aligned}
$$

with (1) defining an isomorphism given by

$$
\left[F^{\cdot}\right] \rightarrow \sum(-1)^{q+l\left(w_{0}\right)}\left[\mathscr{H}^{q} F^{\cdot}\right] .
$$

The groups $K\left(\mathscr{C}_{H, q, \text { mix }}\right)$ have the structure of free $\mathbf{Z}\left[q^{1 / 2}, q^{-1 / 2}\right]$-modules with a basis parametrized by $\mathscr{D}_{H}$. Moreover, they have an additional structure: Denote by $\mathscr{H}_{q}$ the Hecke algebra of $\S 2$ specialized to the specific value $q$. As in [24], $K\left(\mathscr{C}_{H, q, \text { mix }}\right)$ becomes an $\mathscr{H}_{q}$-module. We denote by $\mathscr{M}_{H}$ the corresponding Hecke modules. The action of the operator $T_{s}+1, s$ a simple reflection, can be defined geometrically in a very nice way. Let

$$
\pi_{s}: \mathscr{B}_{q} \rightarrow\left(\mathscr{P}_{s}\right)_{q}
$$

be the projection from the flag variety onto the variety $\left(\mathscr{P}_{s}\right)_{q}$ of parabolic subgroups of $G_{q}$ of type $s$. As in [24] we have

$$
\left(T_{s}+1\right) \gamma=\sum(-1)^{q}\left[\pi_{s}^{*}\left(R^{q} \pi_{s, \gamma}\right)\right] .
$$

Let $\delta, \gamma$ be in $\mathscr{D}_{H}$ and $P_{\gamma, \delta}$ the Kazhdan-Lusztig polynomials as in $\S 2$. Set $\mu(\gamma, \delta)=$ coefficient of $q^{1 / 2(l(\delta)-l(\gamma)-1)}$ in $P_{\gamma, \delta}$. Recall from [24] the definition of the $\tau$-invariant $\tau(\delta)$ and of the set $s \cdot \delta$ associated to $\delta \in \mathscr{D}_{H}$. In $\S 2$, the self-dual elements $\hat{C}_{\delta}$ were introduced. From Lemmas 5.2 and 5.3 of [24], we have formulas for the action of $T_{s}+1$ on $\hat{C}_{\delta}$ :

$$
\begin{array}{ll}
q^{-1 / 2}\left(T_{s}+1\right) \hat{C}_{\delta}=\sum_{\delta^{\prime} \in s \cdot \delta} \hat{C}_{\delta^{\prime}}+\sum_{\gamma \leqslant \delta} \mu(\gamma, \delta) \hat{C}_{\gamma}, & \text { if } s \notin \tau(\delta), \\
q^{-1 / 2}\left(T_{s}+1\right) \hat{C}_{\delta}=\left(q^{1 / 2}+q^{-1 / 2}\right) \hat{C}_{\delta}, & \text { if } s \in \tau(\delta) .
\end{array}
$$


Strong coherence and mixed Jacquet functors. One of the important properties of the usual Jacquet functor $J$ is that it commutes with the functor of tensoring by a finite-dimensional representation. Since we are working in a context where the infinitesimal character is fixed, it is natural to study the $U_{\alpha}$-construction of Vogan [30]. This is very closely connected with the study of the operator $T_{s}+1$ of the Hecke algebra, $s=s_{\alpha}$.

Let $s \in S$ and define the geometric analogue of $U_{\alpha}$,

$$
\tilde{U}_{\alpha}\left(F^{\cdot}\right)=\pi_{s}^{*} \pi_{s !}\left(F^{\cdot}[1]\right) \otimes L^{-1 / 2}
$$

$L=$ trivial sheaf where Fr acts by $q$ (Tate sheaf). By (3.9), $\tilde{U}_{\alpha}$ realizes the operator $-q^{-1 / 2}\left(T_{s_{\alpha}}+1\right)$.

(3.12) Definition. A functor between derived categories of bounded complexes of sheaves

$$
J_{\text {mix }}: D\left(\mathscr{C}_{K, q, \text { mix }}^{\prime}\right) \rightarrow D\left(\mathscr{C}_{N, q, \text { mix }}^{\prime}\right)
$$

will be called a mixed Jacquet functor, if it satisfies:

(M1) (Exactness axiom). $J_{\text {mix }}$ induces an exact functor

$$
J_{\text {mix }}: \mathscr{H}_{\mathscr{C}_{\text {, mix }}} \rightarrow \mathcal{O}_{q, \text { mix }}^{\prime}
$$

(M2) (Strong coherence axiom). There is a natural isomorphism in the derived category

$$
J_{\text {mix }} \tilde{U}_{\alpha} F^{\cdot} \approx \tilde{U}_{\alpha} J_{\text {mix }} F^{\cdot} \text { for } F^{\cdot} \text { in } \mathscr{H} \mathscr{C}_{q, \text { mix }} ;
$$

(M3) (Duality axiom). There is a natural isomorphism

$$
J_{\text {mix }} D F^{\cdot} \approx D J_{\text {mix }} F^{\cdot} \text { for } F^{*} \text { in } \mathscr{H}_{\mathscr{C}_{\text {, mix }}}
$$

(M4) (Support axiom). For any $F^{\cdot}$ in $\mathscr{H} \mathscr{C}_{\mathrm{q} \text {, mix }}$,

$$
\operatorname{dim} \operatorname{support}\left(J_{\text {mix }}\left(F^{\cdot}\right)\right) \leqslant \operatorname{dim} \text { support } F^{*} \text {; }
$$

(M5) (Largest growth axiom). If $\delta \in \mathscr{D}_{K}$ is attached to the open $K_{q}$-orbit, then $J_{\text {mix }}(I C(\delta))$ contáins $1_{\bar{Q}_{1}}$ exactly once as subquotient in $\mathcal{O}_{q \text {,mix }}^{\prime}$, with weight zero.

We have the following proposition relating mixed Jacquet functors and geometric Jacquet functors.

(3.13) Proposition. Let $J_{\text {mix }}$ be a mixed Jacquet functor. Then it induces a geometric Jacquet functor

$$
J_{\text {geo }}: \mathscr{H} \mathscr{C}_{F} \rightarrow \mathcal{O}_{F}^{\prime},
$$

via the equivalences of (3.6) and (3.7).

Proof. The functor $J_{\text {geo }}$ appears from (3.6) and (3.7). Using that $\tilde{U}_{\alpha}$ induces $q^{-1 / 2}\left(T_{s}+1\right)$ on the Hecke modules $\mathscr{M}_{K}, \mathscr{M}_{N}$ (for all but a finite number of characteristics), we satisfy the coherence axiom. A filtration with semisimple subquotients on $J_{\text {geo }}(\bar{\pi}(\delta)), \delta \in \mathscr{D}_{K}$, appears from the weight filtration theorem of Gabber (3.8) and it is designed to realize the weight filtration data from $\mathbf{J}_{\text {geo }}\left(\hat{C}_{\delta}\right)$, as in the definition of a geometric Jacquet functor. Q.E.D.

(3.14) CONJeCture. The usual Jacquet functor defined on $\mathscr{H}_{\mathscr{C}_{F}}$ is induced from a mixed Jacquet functor. 
From (3.13) it is clear that we have

(3.15) Corollary. Conjecture (3.14) implies Conjecture (1.4).

We now devote our efforts to proving the main theorem of this section.

(3.16) THEOREM. There exists a mixed Jacquet functor $J_{\text {mix }}$.

Vanishing cycles construction. The main nontrivial ingredient in our construction of a mixed Jacquet functor $J_{\text {mix }}$ will be the functor of the theory of vanishing cycles $\psi$ of $[2,(4.4)]$. We give a brief description of its construction over $\mathbf{C}$ and refer the reader to [2] for the changes that have to be made to adapt the construction to the field $k_{q}$. In the following we are over $Y^{\text {an }}$.

Let $Y$ be a quasi-projective algebraic variety and $f: Y \rightarrow \mathbf{A}^{1}$ a map of algebraic varieties into the affine line $\mathbf{A}^{1}$. Set $\eta=\mathbf{A}^{1}-(0), Y_{\eta}=f^{-1}(\eta)$. The functor $\psi$ which will be constructed assigns to $F^{*}$, a perverse sheaf on $Y_{\eta}$, a new complex of sheaves over the fiber $Y_{s}=f^{-1}(0)$. Let $\bar{\eta}$ be the universal cover of $\eta$ and $Y_{\bar{\eta}}=Y_{\eta} \times{ }_{\eta} \bar{\eta}$. We have projections

$$
\bar{\eta} \stackrel{P}{\rightarrow} \eta, \quad Y_{\bar{\eta}} \stackrel{P}{\rightarrow} Y_{\eta} .
$$

Setting $\hat{F}=P_{*} P^{*} F^{*}$, a new complex on $Y_{\eta}$ is obtained, which unfortunately does not have constructible cohomology. Let

$$
j: Y_{\eta} \rightarrow Y, \quad i: Y_{s} \rightarrow Y
$$

be the inclusions. We define the complex

$$
\psi\left(F^{*}\right)=i^{*} j_{*}(\hat{F})[-1] .
$$

Over $k_{q}=k$, the only modification is related to the construction of the universal cover $\bar{\eta}$ of $\eta$, which cannot be carried out in the category of algebraic varieties. It has been pointed out to the authors by P. Deligne that the construction of $\psi$ over $\mathbf{C}$ is compatible with the analogous construction over $k_{q}$ for $\overline{\mathbf{Q}}_{\Gamma}$ sheaves, under the equivalence of categories (3.7), but the proof is delicate and is not in the literature. We will not use this compatibility. The following theorem is important for our construction of $J_{\text {mix }}$.

(3.17) THEOREM [2, (4.4)]. Let $Y$ be a quasi-projective algebraic variety over $k, f$ : $Y \rightarrow \mathbf{A}^{1}$ an algebraic map. Let $F^{\cdot}$ be a perverse $\overline{\mathbf{Q}}_{r}$ sheaf on $Y_{\eta}=f^{-1}(\eta)$. Then

(a) $\psi\left(F^{*}\right)$ is perverse.

(b) There is a natural isomorphism $\psi\left(D F^{*}\right) \approx D \psi\left(F^{*}\right)$.

From the definition of $\psi$ in terms of $i^{*} j_{*}$ it is easy to verify that

(3.18) LemMA. If $F^{*}$ is a perverse $\overline{\mathbf{Q}}_{\Gamma}$ sheaf, then

$$
\operatorname{dim} \operatorname{support} \psi\left(F^{\cdot}\right) \leqslant \operatorname{dim} \operatorname{support}\left(F^{\cdot}\right)-1 \text {. }
$$

Proof. We have $\operatorname{dim}$ support $P_{*} P^{*}\left(F^{*}\right)=\operatorname{dim} \operatorname{support}\left(F^{*}\right)$ and the support of $i^{*} j_{*}\left(P_{*} P^{*} F^{*}\right)$ is inside the boundary of the support of $P_{*} P^{*} F^{*}$ in $Y$ consisting of various components of smaller dimension. Q.E.D. 
(3.20) Proposition. Let $\pi: Y_{1} \rightarrow Y_{2}$ be a map of algebraic varieties over $k$. Suppose that $f_{i}: Y_{i} \rightarrow \mathbf{A}^{1}, i=1,2$, are algebraic maps such that $f_{2} \circ \pi=f_{1}$. Then if $\psi_{i}$ denotes the corresponding vanishing cycles functors, $i=1,2$, we have

(a) if $\pi$ is proper, then $\psi_{2} \circ \pi_{*}=\pi_{*} \circ \psi_{1}$,

(b) if $\pi$ is submersive, then $\pi^{*} \circ \psi_{2}=\psi_{1} \circ \pi^{*}$.

PROOF. This is a formal consequence of smooth base change and base change for proper maps [27, (2.3), (4.1)].

$B$-orbits. Let $B, B^{*}$ be opposite Borel subgroups in $G$ and consider the $B$-orbits on $\mathscr{B}$; these coincide with $N$-orbits on $\mathscr{B}$ :

$$
\mathscr{B}_{y}=\{g B, g \in B y B\}, \quad y \in W .
$$

It is well known that $\mathscr{B}_{y}$ is isomorphic to $N \cap y N^{*} y^{-1}$, where $N^{*}$ is the unipotent group "opposite" to $N$. The isomorphism is given by

$$
N \cap y N^{*} y^{-1} \rightarrow \mathscr{B}_{y}, \quad n \rightarrow n y B .
$$

We now review the definition of an affine open neighborhood of $\mathscr{B}_{y}$ inside $\overline{\mathscr{B}}_{\omega}$, following [23]. Consider the varieties

$$
a^{y}=\left\{g B: g B g^{-1} \text { is opposed to } y B^{*} y^{-1}\right\}, \quad \mathscr{B}^{y}=\left\{g B: g \in B^{*} y B\right\} .
$$

Then $a^{y} \cap \overline{\mathscr{B}}_{\omega}$ is an affine open neighborhood of $\mathscr{B}_{y}$ inside $\overline{\mathscr{B}}_{\omega}$. Moreover there is an isomorphism

$$
\begin{aligned}
\mathscr{B}_{y} \times\left(\mathscr{B}^{y} \cap \overline{\mathscr{B}}_{\omega}\right) & \stackrel{\approx}{\rightarrow} \overline{\mathscr{B}}_{\omega} \cap a^{y}, \\
(n y B, g B) & \rightarrow n y B, \quad n \in N \cap y N^{*} y^{-1} .
\end{aligned}
$$

The varieties $\mathscr{B}^{y}$ and $\mathscr{B}_{\omega}$ relate as follows:

$$
y \leqslant \omega \text { (Bruhat order) } \Leftrightarrow \mathscr{B}_{\omega} \cap \mathscr{B}^{y} \neq \varnothing .
$$

We will be interested in the particular case $\omega=s y, s \in S, l(s y)=l(y)+1$ $(l(\omega)=$ the length of $\omega)$. From (3.23), $\mathscr{B}^{y} \cap \overline{\mathscr{B}}_{s y}=\mathscr{B}^{y} \cap\left[\mathscr{B}_{y} \cup \mathscr{B}_{s y}\right]$ which is irreducible of dimension one. Let $N_{s} \subset B, N_{s}^{*} \subset B^{*}$ be the two root groups associated to $s$. We have

(3.24) LEMMA. If $l(s y)=l(y)+1$, with $y \in W, s \in S$, then

$$
N_{s}^{*} \approx \mathscr{B}^{y} \cap \overline{\mathscr{B}}_{s y}=\mathscr{B}^{y} \cap\left[\mathscr{B}_{y} \cup \mathscr{B}_{s y}\right], \quad n \rightarrow n y B .
$$

Proof. Clearly $N_{s}^{*} y B$ is contained in a variety isomorphic to $\mathbf{P}^{1}$, which is the flag variety of a copy of $\mathrm{SL}_{2}$ generated by $N_{s}$ and $N_{s}^{*}$; namely $N_{s}^{*} y B \cup\{s y B\}$. But $s y B \notin \mathscr{B}^{y}$, and the map $N_{s}^{*} \rightarrow \mathscr{B}^{y} \cap \overline{\mathscr{B}}_{s y}$ has to be surjective because $\mathscr{B}^{y} \cap \mathscr{B}_{s y}$ is irreducible of dimension one. Q.E.D.

(3.25) COROLlaRY. There is an affine neighborhood of $\mathscr{B}_{y}$ inside $\mathscr{B}_{y} \cup \mathscr{B}_{s y}$ (where $l(s y)=l(y)+1)$ of the form

$$
\left(\mathscr{B}_{y} \cup \mathscr{B}_{s y}\right) \cap a^{y} \approx \mathscr{B}_{y} \times \mathbf{A}^{1} .
$$


Proof. This follows from the isomorphism in (3.22) and (3.24), noting that the image in (3.22) lies in $\left(\mathscr{B}_{y} \cup \mathscr{B}_{s y}\right) \cap a^{y}$. Q.E.D.

Construction of $J_{\text {mix }}$. We now work over $k=k_{q}$. We have $N_{q} \approx \mathscr{B}_{w_{0}}, w_{0}$ the longest element in $(W, S)$. Consider the maps

$$
\begin{aligned}
N_{q} \times \mathscr{B}_{q} \stackrel{\operatorname{Pr}}{\rightarrow} \mathscr{B}_{q}, & \operatorname{Pr}(u, x)=x, \\
N_{q} \times \mathscr{B}_{q} \stackrel{\varphi}{\rightarrow} N_{q} \times \mathscr{B}_{q}, & \varphi(u, x)=(u, u \cdot x) .
\end{aligned}
$$

If $F^{*}$ is in $\mathscr{H} \mathscr{C}_{q \text {,mix }}$, the new sheaf $\operatorname{Pr}^{*}\left(F^{*}\right)=1_{N} \otimes F^{*}$ has an $N$-action. Let $\Delta$ be the new $N_{q}$-action on $N_{q} \times \mathscr{B}_{q}$ given by $\Delta(n)(u, x)=(n \cdot u, n \cdot x)$, the "diagonal $N$-action" on $N_{q} \times \mathscr{B}_{q}$. The complex of sheaves $\varphi_{*}\left(\operatorname{Pr}^{*}\left(F^{*}\right)\right)$ acquires in this way a $\Delta(N)$-action (see Lemma (3.29)). The idea from here on, in order to produce an object in $\mathcal{O}_{q \text {,mix }}^{\prime}$, is to go from the sheaf $\varphi_{*}\left(\operatorname{Pr}^{*}\left(F^{*}\right)\right)$ on $N_{q} \times \mathscr{B}_{q}$ to a sheaf on $\{e\} \times \mathscr{B}_{q}\left(e \in N_{q}\right.$ the identity) passing through the intermediate stages $N_{q} \cap$ $y N_{q}^{*} y^{-1} \times \mathscr{B}_{q} \approx\left(\mathscr{B}_{y}\right)_{q} \times \mathscr{B}_{q}$. We define

$$
J_{\text {mix }, 0}\left(F^{*}\right)=\varphi_{*} \operatorname{Pr} *\left(F^{*}\right)[\operatorname{dim} N] \otimes L^{-\operatorname{dim} N / 2},
$$

$L=$ Tate sheaf (Fr acts by the scalar $q$ ). Let $w_{0} \in W$ be, as before, the longest element of $(W, S)$ and $w_{0}=s_{1} \cdots s_{k}$ a reduced expression.

Assume inductively that $J_{\text {mix, } r}$ is defined on

$$
\left(\mathscr{B}_{s y}\right)_{q} \times \mathscr{B}_{q}, \quad y=s_{r+1} \cdots s_{1} w_{0}, s=s_{r+1} .
$$

We consider the neighborhood of $\mathscr{B}_{y}$ of the form $\mathscr{B}_{y} \times \mathbf{A}^{1}$ inside $\mathscr{B}_{y} \cup \mathscr{B}_{s y}$ as in (3.25). This gives a projection

$$
f:\left(\mathscr{B}_{y}\right)_{q} \times \mathscr{B}_{q} \times \mathbf{A}^{1} \rightarrow \mathbf{A}^{1}
$$

and it can be assumed that $f^{-1}(0)=\left(\mathscr{B}_{y}\right)_{q} \times \mathscr{B}_{q}$. This defines the functor of vanishing cycles $\psi=\psi_{f}$, and we set

$$
J_{\text {mix }, r+1}\left(F^{\cdot}\right) \stackrel{\text { def }}{=} \psi\left(J_{\text {mix }, r}\left(\left.F^{\cdot}\right|_{f^{-1}(\eta)}\right)\right) .
$$

The complex of sheaves needed is

$$
J_{\text {mix }}\left(F^{\cdot}\right) \stackrel{\text { def }}{=} J_{\text {mix }, \operatorname{dim} N}\left(F^{*}\right)
$$

which lives on $\{e\} \times \mathscr{B}_{q} \approx \mathscr{B}_{q}$.

(3.28) Remark. The composition factors of $J_{\text {mix }}\left(F^{*}\right)$ (moreover its weight filtration!) turn out to be independent of the choice of the reduced expression of $w_{0}$, which was used to "descend" from $N_{q} \times \mathscr{B}_{q}$ to $\mathscr{B}_{q}$ in the construction.

We invite the interested reader to consider the cases $G_{\mathbf{R}}=\mathrm{SL}_{2} \mathbf{R}$ and $G_{\mathbf{R}}=\mathrm{SL}_{2} \mathbf{C}$, where one can see directly how the $K$-orbits "break down" into $N$-orbits, which give the support of the corresponding Jacquet modules. These two simple cases motivated our construction; also see (3.34) below.

We have

(3.29) LemMa. The functor $J_{\text {mix }}$ defined by (3.27) determines an exact functor

$$
J_{\text {mix }}: \mathscr{H}_{\mathscr{C}_{q, \text { mix }}} \rightarrow \mathcal{O}_{q, \text { mix }}^{\prime} .
$$


Proof. Three essential things need to be shown:

(1) $J_{\text {mix }}$ lands in the category of mixed complexes.

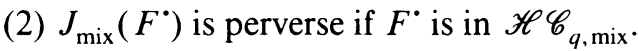

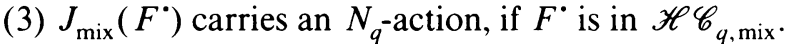

ProOf OF (1). Apply [10, (6.1.11) and (6.1.13)].

ProOF OF (2). We show that $J_{\text {mix, } r}\left(F^{*}\right)$ is perverse by induction on $r$. For $r=0$, $J_{\text {mix }, r}\left(F^{*}\right)=\varphi_{*} \operatorname{Pr}^{*}\left(F^{*}\right)[\operatorname{dim} N] \otimes L^{-\operatorname{dim} N / 2}$ satisfies the perversity conditions (PI) and (PII), as can be seen directly. The condition (PIII) is a consequence of $J_{\text {mix }, 0} D=D J_{\text {mix }, 0}$, which in turn follows from $\varphi_{*}=\varphi_{!}=D \varphi_{*} D$ and $\operatorname{Pr} !=$ $\operatorname{Pr}^{*}[2 \operatorname{dim} N] \otimes L^{-\operatorname{dim} N}=D \operatorname{Pr}^{*} D$. We now assume the perversity of $J_{\text {mix, }}\left(F^{*}\right)$, to obtain that $J_{\text {mix }, r+1}\left(F^{*}\right)$ is perverse (by (3.17)), since

$$
J_{\text {mix }, r+1}\left(F^{\cdot}\right)=\psi\left(\left.J_{\text {mix }, r}\left(F^{\cdot}\right)\right|_{f^{-1}(\eta)}\right) .
$$

Proof OF (3). We have two actions of $N_{q}$ on $N_{q} \times \mathscr{B}_{q}$ that we want to consider: the "straight" $N_{q}$-action given by $n \cdot(x, y)=(n \cdot x, y)$; and the "diagonal" action, given by $\Delta(n)(x, y)=(n \cdot x, n \cdot y)$. Similarly, we have two actions at each of the intermediate stages $\left(\mathscr{B}_{y}\right)_{q} \times \mathscr{B}_{q}$. Consider the maps $N_{q} \times N_{q} \times \mathscr{B}_{q} \rightarrow N_{q}$ $\times \mathscr{B}_{q}$, given by $p(x, y, z)=(y, z)$, act $(x, y, z)=(x \cdot y, z)$, and $\operatorname{act}_{\Delta}(x, y, z)=$ $(x \cdot y, x \cdot z)$. The sheaf $\operatorname{Pr}^{*}\left(F^{*}\right)=1_{N_{q}} \otimes F^{*}$ has a "straight" $N_{q}$-action: There is an isomorphism

$$
\alpha: p^{*}\left(1_{N_{q}} \otimes F^{*}\right) \rightarrow \operatorname{act} *\left(1_{N} \otimes F^{*}\right)
$$

which restricted to $\{e\} \times N_{q} \times \mathscr{B}_{q}$ ( $e$ the identify of $N_{q}$ ) is the identity between $1_{N} \otimes F^{\cdot}$ and itself. We use that $\varphi^{-1} \circ$ act $_{\Delta}=\operatorname{act} \circ\left(1 \times \varphi^{-1}\right)$, with $(1 \times \varphi)(x, y, z)=$ $(x, y, y \cdot z)$, to write $\operatorname{act}_{\Delta}^{*}\left(\varphi_{*} \operatorname{Pr}^{*} F^{*}\right) \approx\left(1 \times \varphi^{-1}\right)^{*} \operatorname{act}^{*}\left(\operatorname{Pr}^{*} F^{*}\right)$. Then, the isomorphism $\alpha$ gives an isomorphism

$$
\left(1 \times \varphi^{-1}\right)^{*} p^{*} \operatorname{Pr}^{*} F^{*} \rightarrow\left(1 \times \varphi^{-1}\right)^{*} \operatorname{act}^{*} \operatorname{Pr}^{*} F^{*} ;
$$

but $p \circ\left(1 \times \varphi^{-1}\right)=\varphi^{-1} \circ p \Rightarrow p^{*} \varphi_{*}=\left(1 \times \varphi^{-1}\right)^{*} p^{*}$ and we obtain an isomorphism:

$$
p^{*}\left(J_{\text {mix }, 0}\left(F^{*}\right)\right) \rightarrow \operatorname{act}_{\Delta}^{*}\left(J_{\text {mix }, 0}\left(F^{*}\right)\right)
$$

defining a $\Delta(N)$-action on $J_{\text {mix }, 0}\left(F^{*}\right)$.

By induction, we assume that $J_{\text {mix, } r}\left(F^{*}\right)=S^{*}$ carries a $\Delta\left(N_{q}\right)$-action over $\left(\mathscr{B}_{s y}\right)_{q}$ $\times \mathscr{B}_{q}, y=s_{r+1} \cdots s_{1} w_{0}, s=s_{r+1}$. Recall the neighborhood of $\left(\mathscr{B}_{y}\right)_{q}$ of the form $\left(\mathscr{B}_{y}\right)_{q} \times \mathbf{A}^{1}$ inside $\left(\mathscr{B}_{y}\right)_{q} \cup\left(\mathscr{B}_{s y}\right)_{q}$. As before, we have maps $N_{q} \times\left(\left(\mathscr{B}_{y}\right)_{q} \cup\right.$ $\left.\left(\mathscr{B}_{s y}\right)_{q}\right) \times \mathscr{B}_{q} \rightarrow\left(\left(\mathscr{B}_{y}\right)_{q} \cup\left(\mathscr{B}_{s y}\right)_{q}\right) \times \mathscr{B}_{q}, \quad p(n, x)=x, \operatorname{act}_{\Delta}(n, x)=\Delta(n) \cdot x$. By induction, there is an isomorphism

$$
\alpha^{\prime}: p^{*}\left(S^{\cdot}\right) \rightarrow \operatorname{act}_{\Delta}^{*}\left(S^{*}\right)
$$

which restricts to the identity between $S^{\cdot}$ and itself on $\{e\} \times\left(\mathscr{B}_{s y}\right)_{q} \times N_{q}$. Let $L=\left(\mathscr{B}_{y}\right)_{q} \times \mathbf{A}^{1} \times \mathscr{B}_{q}$, and $L^{\prime}=\operatorname{act}_{\Delta}^{-1}(L) \cap p^{-1}(L)$. Since $L^{\prime} \subset N_{q} \times L$ which maps onto $\mathbf{A}^{1}$, we obtain a map $f^{\prime}: L^{\prime} \rightarrow \mathbf{A}^{1}$, defining a new vanishing cycles functor $\psi_{f^{\prime}}$ on $L^{\prime}$. 
By restricting $\alpha^{\prime}$ to $L^{\prime}$, we obtain an isomorphism

$$
\alpha^{\prime \prime}: \psi_{f^{\prime}}\left(\left.p^{*}\left(S^{\bullet}\right)\right|_{L_{\eta}^{\prime}}\right) \rightarrow \psi_{f^{\prime}}\left(\left.\operatorname{act}_{\Delta}^{*}\left(S^{\bullet}\right)\right|_{L_{\eta}^{\prime}}\right) .
$$

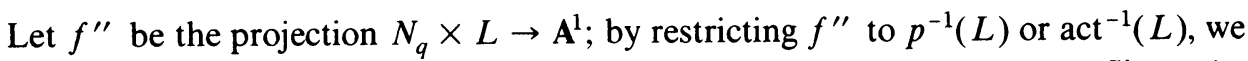
obtain two vanishing cycles functors which we simply denote as $\psi_{f^{\prime \prime}}$. Since $\psi_{f^{\prime}}$ commutes with restriction to open subsets,

$$
\begin{aligned}
\psi_{f^{\prime}}\left(\left.p^{*}\left(S^{*}\right)\right|_{L_{\eta}^{\prime}}\right) & \approx \psi_{f^{\prime \prime}}\left(\left.p^{*}\left(S^{\bullet}\right)\right|_{p^{-1}(L)_{\eta}}\right), \\
\psi_{f^{\prime}}\left(\left.\operatorname{act}_{\Delta}^{*}\left(S^{\bullet}\right)\right|_{L_{\eta}^{\prime}}\right) & \approx \psi_{f^{\prime \prime}}\left(\left.\operatorname{act}_{\Delta}^{*}\left(S^{*}\right)\right|_{\operatorname{act}^{-1}(L)_{\eta}}\right) .
\end{aligned}
$$

But, by (3.20), and the fact that act factors as $\operatorname{act}_{\Delta}=p \circ \varphi^{\prime}, \varphi^{\prime}(x, y, z)=$ $(x, x \cdot y, x \cdot z)$, with $p$ submersive and $\varphi^{\prime}$ proper, we obtain

$$
\begin{aligned}
\psi_{f^{\prime \prime}}\left(\left.p^{*}\left(S^{*}\right)\right|_{p^{-1}(L)_{\eta}}\right) & \approx p^{*} \psi\left(\left.S^{*}\right|_{L_{\eta}}\right), \\
\psi_{f^{\prime \prime}}\left(\left.\operatorname{act}_{\Delta}^{*}\left(S^{*}\right)\right|_{\operatorname{act}^{-1}\left(L_{\eta}\right)}\right. & \approx \operatorname{act}_{\Delta}^{*} \psi\left(\left.S^{*}\right|_{L_{\eta}}\right) .
\end{aligned}
$$

Hence, we obtain an isomorphism, which defines a "diagonal" action on $J_{\text {mix }, r+1}\left(F^{*}\right)$

$$
\alpha^{\prime \prime \prime}: p^{*}\left(\psi\left(\left.S^{*}\right|_{L_{\eta}}\right)\right) \rightarrow \operatorname{act}_{\Delta}^{*}\left(\psi\left(\left.S^{\cdot}\right|_{L_{\eta}}\right)\right) .
$$

It remains to prove that $\alpha^{\prime \prime \prime}$ is the identity on $\{e\} \times\left(\mathscr{B}_{s y}\right)_{q} \times \mathscr{B}_{q}$.

Let $s:\{e\} \times\left(\mathscr{B}_{y}\right)_{q} \times \mathscr{B}_{q} \rightarrow N_{q} \times\left(\mathscr{B}_{y}\right)_{q} \times \mathscr{B}_{q}$ be the inclusion. Note that $p \circ s$ $=\operatorname{act}_{\Delta} \circ s=$ identity, and we have base-change maps defined as in [27, p. 223], and a commutative diagram:

$$
\begin{array}{ccc}
\psi\left(s^{*} p^{*}\left(\left.S^{\cdot}\right|_{L_{\eta}}\right)\right) & \stackrel{1}{\leftarrow} & s^{*} p^{*} \psi\left(\left.S \cdot\right|_{L_{\eta}}\right) \\
\text { (3) } \downarrow & & \downarrow \text { (4) } \\
\psi\left(s^{*} \operatorname{act}_{\Delta}^{*}\left(\left.S^{*}\right|_{L_{\eta}}\right)\right) & \stackrel{2}{\leftarrow} & s^{*} \operatorname{act}_{\Delta}^{*} \psi\left(\left.S \cdot\right|_{L_{\eta}}\right)
\end{array}
$$

Here (1) and (2) are in the class of the identity and (4) will be the identity if (3) is the identity. The map (3) is the identity by the induction hypothesis.

Finally, since the "diagonal" and "usual" actions coincide on $\{e\} \times \mathscr{B}_{q}$, we obtain the desired $N_{q}$-action on $J_{\text {mix }}\left(F^{*}\right)$ ! Q.E.D.

(3.30) Lemma. For any $F^{*}$ in $\mathscr{H}_{\mathscr{C}_{\text {, mix }}}$ there is an isomorphism

$$
J_{\text {mix }}\left(D F^{\cdot}\right) \approx D J_{\text {mix }}\left(F^{\cdot}\right) \text {. }
$$

Proof. This follows from (3.17)(b), i.e., the fact that $\psi$ commutes with $D$. All the shifts in the definition were designed to insure the other functors involved commute with D. Q.E.D.

(3.31) LEMMA. The functor $J_{\text {mix }}$ constructed above commutes with $\tilde{U}_{\alpha}\left(s=s_{\alpha} \in S\right)$. 
ProOF. We have a commutative diagram:

$$
\begin{array}{ccc}
N_{q} \times \mathscr{B}_{q} & \stackrel{\varphi}{\rightarrow} & N_{q} \times \mathscr{B}_{q} \\
1 \times \pi_{s} \downarrow & \downarrow 1 \times \pi_{s} \\
N_{q} \times\left(\mathscr{P}_{s}\right)_{q} & \stackrel{\varphi}{\rightarrow} & N_{q} \times \mathscr{B}_{q}
\end{array}
$$

Hence $\left(1 \times \pi_{s}\right)_{*} \varphi_{*}=\varphi_{*}\left(1 \times \pi_{s}\right)_{*}$, and by base-change $\varphi_{*}\left(1 \times \pi_{s}\right)^{*}=\left(1 \times \pi_{s}\right)^{*} \varphi_{*}$. Thus,

$$
J_{\text {mix }, 0}\left(1 \times \pi_{s}^{*}\right)=\left(1 \times \pi_{s}^{*}\right) J_{\text {mix }, 0} .
$$

Assume inductively that $J_{\text {mix, } r}$ commutes with $\left(1 \times \pi_{s}\right)^{*}$ and $\left(1 \times \pi_{s}\right)_{*}$. Recall that

$$
J_{\text {mix }, r+1}=\psi J_{\text {mix }, r} .
$$

Using that $\psi$ commutes with $\left(1 \times \pi_{s}\right)_{*}$ and $\left(1 \times \pi_{s}\right)^{*}$ (because $1 \times \pi_{s}$ is proper and submersive), we conclude that $J_{\text {mix, } r}$ commutes with $\left(1 \times \pi_{s}\right)_{*},\left(1 \times \pi_{s}\right)^{*}$. By setting $r=\operatorname{dim} \mathscr{B}$, we conclude that $J_{\text {mix }}$ commutes with $\tilde{U}_{\alpha}$. Q.E.D.

(3.32) LemMa. The functor $J_{\text {mix }}$ constructed above satisfies the support condition

$$
\operatorname{dim} \operatorname{support} J_{\text {mix }}\left(F^{*}\right) \leqslant \operatorname{dim} \operatorname{support}\left(F^{*}\right) \text {. }
$$

Proof. This is a simple consequence of (3.18), since

$$
\operatorname{dim} \operatorname{support} \psi\left(J_{\text {inix }, r}\left(F^{\cdot}\right)\right) \leqslant \operatorname{dim} \operatorname{support} J_{\text {mix }, r}\left(F^{\cdot}\right)-1
$$

and by induction,

$$
\operatorname{dim} \text { support } J_{\text {mix }, r}\left(F^{*}\right) \leqslant \operatorname{dim} \operatorname{support} F^{\cdot}+\operatorname{dim} \mathscr{B}-r . \quad \text { Q.E.D. }
$$

(3.33) Lemma. The functor $J_{\mathrm{mix}}$ satisfies the largest growth axiom, i.e., if $F^{*}$ is irreducible and attached to an open $K_{q}$-orbit, then $J_{\text {mix }}\left(F^{*}\right)$ contains the trivial $\overline{\mathbf{Q}}_{\Gamma}$ sheaf exactly once as subquotient with weight zero.

Proof. For complex groups (or groups with connected Cartan subgroups), the only possibility is that $F^{\cdot}$ be a trivial sheaf. By Corollary 2.4 of [15], $\psi$ takes a trivial sheaf to be a trivial sheaf when $f$, the map which is used to define $\psi$, is submersive. For the general case, a simple modification of the argument in [15] shows that if $K^{\circ}$ is a perverse sheaf which is locally constant with one-dimensional stalks on an open set, $\psi\left(K^{*}\right)$ will also be locally constant with one-dimensional stalks on a Zariski open subset. By induction, we obtain that $J_{\text {mix }}\left(F^{*}\right)$ has to be a trivial sheaf on $\mathscr{B}_{w_{0}}$, thus a trivial sheaf arises exactly once as a subquotient and the weight zero statement follows, from the fact that $J_{\text {mix }}\left(F^{*}\right)$ is self-dual. Q.E.D.

(3.34) More on $\mathrm{SL}_{2} \mathbf{R}$. We now go back to our example in (2.11), where $G_{\mathbf{R}}=$ $\mathrm{SL}_{2} \mathbf{R}$. As before, $\delta_{-}$is the irreducible principal series, and we have computed $\mathbf{J}_{\text {geo }}\left(\hat{C}_{\delta_{-}}\right)=-q^{-1 / 2} \hat{C}_{e}+q^{0} \hat{C}_{s}-q^{1 / 2} \hat{C}_{e}$, corresponding to a weight filtration of $J_{\text {geo }}\left(\bar{\pi}\left(\delta_{-}\right)\right)$,

$$
L_{e}<L_{s}<L_{e} .
$$


We will now show that, in fact, there are nontrivial extensions between consecutive weight levels. It is convenient to alter the realization $G_{\mathbf{R}}=\operatorname{SU}(1,1)$ (as in (2.11)), so that

$$
N_{q}=\left\{\left(\begin{array}{cc}
1 & t \\
0 & 1
\end{array}\right), t \in k\right\} \approx \mathbf{A}^{1}
$$

inside $\mathrm{SL}_{2} k$. The flag variety is $\mathscr{B}_{q}=\mathbf{P}^{1}$ and the $N_{q}$-orbits become $\{\infty\}$ and $\mathbf{P}^{1}-\{\infty\}$. The $K_{q}$-orbits are $\{x\},\{y\}$, and $\mathbf{P}^{1}-\{x, y\}, x \neq y$.

As in the construction of $J_{\text {mix }}$, we have maps

$$
\begin{gathered}
\operatorname{Pr}: N_{q} \times \mathbf{P}^{1} \rightarrow \mathbf{P}^{1}, \quad(t, z) \rightarrow z, \\
\varphi: N_{q} \times \mathbf{P}^{1} \rightarrow N \times \mathbf{P}^{1}, \quad(t, z) \rightarrow(t, z+t) .
\end{gathered}
$$

Recall $J_{\text {mix }, 0}=\varphi_{*} \operatorname{Pr}{ }^{*}[1] \otimes L^{-1 / 2}$. Hence $J_{\text {mix }, 0}$ applied to $\delta_{a}, \delta_{h}, \delta_{+}, \delta_{-}$, consists, respectively, of a local system extended by zero over the varieties

$$
\begin{array}{ll}
Z_{a}=\{(t, t+x), t \in k\} & \text { for } \delta_{a}, \\
Z_{h}=\{(t, t+y), t \in k\} & \text { for } \delta_{h}, \\
Z=N_{q} \times \mathbf{P}^{1}-\left(Z_{a} \cup Z_{h}\right) & \text { for } \delta_{+} \text {and } \delta_{-} .
\end{array}
$$

The affine neighborhood of $\{\infty\}$ used to define $\psi$ is $\mathbf{P}^{1}-\{0\}$ and $f$ is given by

$$
U=\left(\mathbf{P}^{1}-\{0\}\right) \times \mathbf{P}^{1} \stackrel{f}{\rightarrow} \mathbf{A}^{1}, \quad(t, z) \rightarrow 1 / t,
$$

so that $f^{-1}(0)=\infty \times \mathbf{P}^{1}$. This function $f$ defines $\psi$ and, as before, $J_{\text {mix }}=$ $\psi\left(\left.J_{\text {mix }, 0}\right|_{U}\right)$. The supports for the components of $J_{\text {mix }}$ are then

$$
\begin{aligned}
& J_{\text {mix }}\left(\delta_{a}\right) \leftrightarrow \bar{Z}_{a} \cap \infty \times \mathbf{P}^{1}=\{(\infty, \infty)\}, \\
& J_{\text {mix }}\left(\delta_{h}\right) \leftrightarrow \bar{Z}_{h} \cap \infty \times \mathbf{P}^{1}=\{(\infty, \infty)\}, \\
& J_{\text {mix }}\left(\delta_{ \pm}\right) \leftrightarrow \bar{Z} \cap \infty \times \mathbf{P}^{1}=\infty \times \mathbf{P}^{1} .
\end{aligned}
$$

The first two are, of course, the supports of $L_{e}=J_{\text {geo }}\left(\delta_{a}\right)=J_{\text {geo }}\left(\delta_{h}\right)$; the last one corresponds to the support of $J_{\text {geo }}\left(\delta_{+}\right)$or of $J_{\text {geo }}\left(\delta_{-}\right)$, which we now study in more detail. For convenience, we consider everything over $\mathbf{C}$ from now on.

$J_{\text {mix }, 0}\left(\delta_{-}\right)$is a nontrivial local system $\mathscr{L}$ over $N_{q} \times \mathbf{P}^{1}-\left(Z_{a} \cup Z_{h}\right)$, extended by zero. Since $\bar{Z}_{a}$ and $\bar{Z}_{h}$ meet at $(\infty, \infty)$, around each point $z \in \infty \times \mathbf{P}^{1}-\{(\infty, \infty)\}$, we can consider the neighborhood

$$
\left(\mathbf{P}^{1}-\{0\}\right) \times W \approx \mathbf{A}^{1} \times W
$$

with $W$ a small disc inside $\mathbf{P}^{1}, z \in W$. If $P: \bar{\eta} \rightarrow \eta=\mathbf{P}^{1}-\{0, \infty\}$ is the universal cover, we obtain

$$
\begin{aligned}
\mathscr{H}^{i}\left(J_{\text {mix }}\left(\delta_{-}\right)[1]\right)_{z} & =H^{i}\left(\bar{\eta} \times W, P^{*} \mathscr{L}\right)=H^{i}(\bar{\eta} \times W, \mathbf{C}) \\
& = \begin{cases}0, & i \neq 0, \\
\mathbf{C}, & i=0 .\end{cases}
\end{aligned}
$$


Over $Z_{a} \cup Z_{h}, J_{\text {mix }, 0}\left(\delta_{-}\right)$is zero, thus we obtain $P_{*} P^{*}\left(\left.J_{\text {mix }, 0}\left(\delta_{-}\right)\right|_{U_{n}}\right)$ is zero on $Z_{a} \cup Z_{h}$ and so is $\mathscr{H}^{0}\left(J_{\text {mix }}\left(\delta_{-}\right)[1]\right)=\mathscr{H}^{0} i^{*} j_{*} P_{*} P^{*}\left(\left.J_{\text {mix }, 0}\left(\delta_{-}\right)\right|_{u_{\eta}}\right)$. Hence, $\mathscr{H}^{0} J_{\text {mix }}\left(\delta_{-}\right)$is a local system on $\infty \times \mathbf{P}^{1}-\{\infty, \infty\}$ extended to zero. This is the perverse sheaf associated to the Verma module containing $F$ as a quotient. Hence, we obtain a nontrivial extension between two of the weight levels, and by duality, we have nontrivial extensions between all the weight levels. The conclusion is that if $J_{\text {geo }}$ is induced from $J_{\text {mix }}$,

$$
L_{e}<L_{s}<L_{e}
$$

is the socle filtration of $J_{\text {mix }}\left(\delta_{-}\right)$. Note that with our conventions, the irreducible principal series corresponds to $\delta_{-}[1]$ rather than to $\delta_{-}$, and this shifts the answers in the above calculation.

4. Special cases of the conjecture. Throughout this section, until further notice, $G_{\mathbf{R}}$ will denote a connected simple real rank one matrix group. Our goal is to establish the following strengthened version of (1.4).

(4.1) THEOREM. In the case of $\mathbf{R}$-rank one, the weight filtration of $\mathbf{J}_{\text {geo }}\left(\hat{C}_{\delta}\right)$ $\left(\delta \in \mathscr{D}_{K}\right)$ corresponds to the socle filtration of $J(\bar{\pi}(\delta))$.

Our proof of (4.1) depends upon case-by-case analysis, corresponding to the classification of $G_{\mathbf{R}}$, being covered by $\operatorname{Spin}(n, 1), \operatorname{SU}(n, 1), \operatorname{Sp}(n, 1)$, or $F_{4(-20)}$. Moreover, our argument is in some sense indirect. That is, we will first compute the weight (Frobenius) filtration of $\mathbf{J}_{\text {geo }}\left(\hat{C}_{\delta}\right)$, using the algorithm of $\S 2$. Next, using totally different means, we compute the socle filtration $J(\bar{\pi}(\delta))$. In the end, we compare our two "lists" and see things match up as asserted.

A certain amount of notation (mainly indexing) is necessary, with which we must assume full familiarity. These assumptions (with references) are summarized below. Also, we assume $G \neq \mathrm{SL}_{2} \mathbf{R}$. This is no loss of generality, in view of the example discussed in (2.11).

(4.2) Orbit structures. Before proceeding, we need to parametrize $\mathscr{D}_{K}$ and $\mathscr{D}_{N}$. First, consider the $K$-orbits. Since $M_{m, \mathbf{R}}$ is connected $\left(G \neq \mathrm{SL}_{2} \mathbf{R}\right.$ !), this is an infinitesimal problem; i.e., we need to parametrize certain positive root systems for $\Phi$ and certain positive root systems for the roots attached to a compact Cartan subalgebra (if such exists). Recall, $G_{\mathbf{R}}$ has a compact Cartan subgroup (equivalently, $G_{\mathbf{R}}$ has a discrete series) if and only if $G_{\mathbf{R}} \neq \operatorname{Spin}(2 n+1,1)$ [29]. All such positive systems are in correspondence with their associated half-sums of positive roots. For the positive systems attached to $\Phi$, we use indexing $\gamma_{i j}$ and for the positive systems attached to the discrete series, we use $\gamma_{i}$. The exact indexing is given in [7, 9, or 29], specific to each case for $G_{\mathbf{R}}$. To each $\gamma_{i j}, \gamma_{k}$ we attach standard representations $\pi_{i j}$, $\pi_{k}$, and their unique irreducible quotients $\bar{\pi}_{i j}, \bar{\pi}_{k}$.

Next, we need to consider $\mathscr{D}_{N}$. At this point, it is extremely fruitful to make a simplifying assumption. Define

$$
\text { category } \mathcal{O}_{F, m}^{\prime}=\text { modules in } \mathcal{O}_{F} \text { which are } \mathfrak{p}_{m} \text {-finite. }
$$


Then $\mathcal{O}_{F, m}^{\prime}$ is a subcategory of $\mathcal{O}_{F}^{\prime}$ and our functors $J_{\text {geo }}$ and $J$ actually take values in here. (The fact that $J$ takes values in here is contained in [5, 28, or 37]. By (2.26), the same statement holds for $J_{\text {geo }}$.) This observation is useful in the sense that $\mathscr{D}\left(\mathcal{O}_{F, m}^{\prime}\right) \stackrel{\text { def }}{=} \mathscr{D}_{N, m}$ is a "smaller set." Namely, it will be in one-to-one correspondence with $W^{m}=W_{m} \backslash W ; W_{m}=$ Weyl group of $\mathfrak{h}_{m}$ in $\mathfrak{m}_{m} \oplus \mathfrak{a}_{m}$ (the $m$-Weyl group). We will identify $W^{m}$ with minimal length left coset representations, and in this sense $W^{m}$ “ $\subset$ " $W$. Using such representatives, call them $w_{t}$ 's, we can explicitly write out the highest weights of the irreducible objects $L_{t}$ in category $\mathcal{O}_{F, m}^{\prime}$. (They are the unique irreducible quotients of the generalized Verma modules $V_{w_{t}}=\mathfrak{U}(\mathfrak{g}) \otimes_{\mathfrak{p}_{m}} E_{t}$, $\sigma_{t}$ on $E_{t}$ ranging over the appropriate irreducible $\mathfrak{m}_{m} \oplus \mathfrak{a}_{m}$-modules. This is all fully discussed in [3].) In the end, we have parameters $w_{i j}$ and $w_{i j^{j}}$ for $W^{m}$, as in Figures 1-4 of [3]. (The " $\theta$ " superscript will play no role here, but it would only add confusion to change the notation.) It is worth noting that $w_{01}$ is always attached to $F$ and $w_{01^{\theta}}$ corresponds to the irreducible generalized Verma module in $\mathcal{O}_{F, m}^{\prime}$.

With $\mathscr{D}_{K}$ and $\mathscr{D}_{N, m}$ parametrized, we must understand the containment relation among closures of $K$ and $P_{m}$ orbits on $\mathscr{B}$. Figures 1-4 of [3] are precisely these relations for $P_{m}$-orbits: the containment relation is generated by the line segments (see Figure 5 for $\mathrm{SU}(4,1)$ ). The $K$-orbit pictures are given in Tables 1-4 of [7]. Unfortunately, we did not include the containment relations, but these can be easily gleaned by superimposing the $K$ picture onto the upper half of the corresponding $P_{m}$ picture, forgetting the $\bar{\pi}_{i}$-row. For that row (which corresponds to the discrete series), we use Cayley transforms to generate the containment relations; e.g., see Figure 5. Alternatively, see [9, (4.5)].

We now present the main results leading to (4.1). Remarks concerning their proofs are given below. Also, we adhere to the notation of (2.8), to indicate levels in the socle filtration or levels in the weight filtration.

(4.3) Proposition. If $G_{\mathbf{R}}=\operatorname{Spin}(n, 1)$, then the weight filtration of $\mathbf{J}_{\text {geo }}\left(\hat{C}_{\gamma}\right)$, $\gamma \in \mathscr{D}_{K}$, corresponds to the socle filtration of $J(\bar{\pi}(\gamma))$ and both are given as follows:

(i) $n=2 k+1$ :

$$
\begin{aligned}
& J\left(\bar{\pi}_{01}\right)=L_{01}, \\
& J\left(\bar{\pi}_{0 i}\right)=L_{(0, i-1)^{\theta}}<L_{0 i}<L_{(0, i-1)^{\theta}}, \quad 2 \leqslant i \leqslant k, \\
& J\left(\bar{\pi}_{0, k+1}\right)=L_{(0, k)^{\theta}}<L_{0, k+1} \oplus L_{(0, k+1)^{\theta}}<L_{(0, k)^{\theta}} .
\end{aligned}
$$

(ii) $n=2 k$ :

$$
\begin{aligned}
& J\left(\bar{\pi}_{01}\right)=L_{01}, \\
& J\left(\bar{\pi}_{0}\right)=J\left(\bar{\pi}_{1}\right)=L_{(0, k)^{\theta},} \\
& J\left(\bar{\pi}_{0 i}\right)=L_{0 i} \oplus L_{(0, i-1)^{\theta}}, \quad 2 \leqslant i \leqslant k .
\end{aligned}
$$

(4.4) Proposition. If $G_{\mathbf{R}}=\mathrm{SU}(n, 1)$, then the weight filtration of $\mathbf{J}_{\text {geo }}\left(\hat{C}_{\gamma}\right), \gamma \in \mathscr{D}_{K}$, corresponds to the socle filtration of $J(\bar{\pi}(\gamma))$ and both are given as follows:

(i) $0 \leqslant i \leqslant n-1, J\left(\bar{\pi}_{i 1}\right)=L_{i 1}$,

(ii) $1 \leqslant i \leqslant n, J\left(\bar{\pi}_{0 i}\right)=L_{0 i}$,

(iii) $J\left(\bar{\pi}_{0}\right)=L_{(0, n)^{\theta}}$, 
(iv) $J\left(\bar{\pi}_{n}\right)=L_{(n-1,1)^{\theta}}$,

(v) for all remaining possible $(i, j)$ indices,

$$
J\left(\bar{\pi}_{i j}\right)=L_{(i-1, j-1)^{\theta}}<L_{i j}<L_{(i-1, j-1)^{\theta}},
$$

(vi) $1 \leqslant i \leqslant n-1$,

$$
J\left(\bar{\pi}_{i}\right)=L_{(i-1, n-i)^{\theta}}<L_{(i-1, n-i+1)^{\theta}} \oplus L_{(i, n-i)^{\theta}}<L_{(i-1, n-i)^{\theta}} .
$$

(4.5) Proposition. If $G_{\mathbf{R}}=\operatorname{Sp}(n, 1)$, then the weight filtration of $\mathbf{J}_{\mathrm{geo}}\left(\hat{C}_{\gamma}\right), \gamma \in \mathscr{D}_{K}$, corresponds to the socle filtration of $J(\bar{\pi}(\gamma))$ and both are given as follows:

(i) $1 \leqslant i \leqslant 2 n, J\left(\bar{\pi}_{0 i}\right)=L_{0 i}$,

(ii) $J\left(\bar{\pi}_{12}\right)=L_{12}$,

(iii) $J\left(\bar{\pi}_{0}\right)=L_{(0,2 n)^{\theta}}$,

(iv) $J\left(\bar{\pi}_{n-1, n+1}\right)=L_{(n-2, n)^{\theta}}<L_{n-1, n+1} \oplus L_{(n-1, n)^{\theta}}<L_{(n-2, n)^{\theta}}$,

(v) for all remaining $(i, j)$ indices,

$$
J\left(\bar{\pi}_{i j}\right)= \begin{cases}L_{(i-1, j-1)^{\theta}}<L_{i j}<L_{(i-1, j-1)^{\theta}}, & i \neq j-1, \\ L_{(i-2, j-2)^{\theta}}<L_{i j}<L_{(i-2, j-2)^{\theta}}, & i=j-1,\end{cases}
$$

(vi) $J\left(\bar{\pi}_{n}\right)=L_{(n-2, n-1)^{\theta}}<L_{(n-1, n+1)^{\theta}}<L_{(n-2, n-1)^{\theta}}$,

(vii) $1 \leqslant i \leqslant n-1$,

$$
J\left(\bar{\pi}_{i}\right)=L_{(i-1,2 n-i)^{\theta}}<L_{(i-1,2 n-i+1)^{\theta}} \oplus L_{(i, 2 n-i)^{\theta}}<L_{(i-1,2 n-i)^{\theta}} .
$$

(4.6) Proposition. If $G_{\mathbf{R}}=F_{4(-20)}$, then the weight filtration of $\mathbf{J}_{\text {geo }}\left(\hat{C}_{\gamma}\right), \gamma \in \mathscr{D}_{K}$, corresponds to the socle filtration of $J(\bar{\pi}(\gamma))$ and both are given as follows:

$$
\begin{aligned}
& J\left(\bar{\pi}_{01}\right)=L_{01}, \quad J\left(\bar{\pi}_{05}\right)=L_{05}, \\
& J\left(\bar{\pi}_{02}\right)=L_{02}, \quad J\left(\bar{\pi}_{14}\right)=L_{14}, \\
& J\left(\bar{\pi}_{03}\right)=L_{03}, \quad J\left(\bar{\pi}_{24}\right)=L_{24}, \\
& J\left(\bar{\pi}_{04}\right)=L_{04}, \quad J\left(\bar{\pi}_{25}\right)=L_{14^{\theta}}<L_{24^{\theta}} \oplus L_{25}<L_{14^{\theta}}, \\
& J\left(\bar{\pi}_{26}\right)=L_{15^{\theta}}<L_{26} \oplus L_{25^{\theta}}<L_{15^{\theta}}, \\
& J\left(\bar{\pi}_{15}\right)=L_{04^{\theta}}<L_{15}<L_{04^{\theta}}, \\
& J\left(\bar{\pi}_{16}\right)=L_{03^{\theta}}<L_{16}<L_{03^{\theta}}, \\
& J\left(\bar{\pi}_{17}\right)=L_{02^{\theta}}<L_{17}<L_{02^{\theta}}, \\
& J\left(\bar{\pi}_{3}\right)=L_{01^{\theta}}<L_{17^{\theta}}<L_{01^{\theta}}, \\
& J\left(\bar{\pi}_{2}\right)=L_{16^{\theta}}<L_{17^{\theta}} \oplus L_{26^{\theta}}<L_{16^{\theta}}, \\
& J\left(\bar{\pi}_{1}\right)=L_{05^{\theta}}<L_{26^{\theta}}<L_{05^{9}} .
\end{aligned}
$$

(4.7) Remark. The structure of $J(\bar{\pi}(\gamma))$ is intimately connected with the zero $\bar{n}$-homology of $\bar{\pi}(\gamma)$, as is computed in [7]. If $J(\bar{\pi}(\gamma))$ is irreducible, then $\bar{\pi}(\gamma)$ has irreducible zero $\overline{\mathfrak{n}}$-homology; such modules are termed unique embedding modules in [6]. If $J(\bar{\pi}(\gamma))$ has two irreducible composition factors (which only happens in the Spin $(2 n, 1)$ case), then $J(\bar{\pi}(\gamma))$ is semisimple; this was noted in [6]. If $J(\bar{\pi}(\gamma))$ has three irreducible composition factors, then $H_{0}(\bar{n}, \bar{\pi}(\gamma))$ is two dimensional. Finally, the case when $J(\bar{\pi}(\gamma))$ has four irreducible constituents is precisely the case when $H_{0}(\bar{n}, \bar{\pi}(\gamma))$ is three dimensional. These connections are all schematically codified in Tables $1-4$ of [7]. 
The proofs of (4.3)-(4.6) are completely straightforward verifications-albeit tedious - which are substantially left to the reader. We will sketch the essential ideas.

Proofs of (4.3)-(4.6): Geometric PART. Replace $\bar{\pi}_{i j}$ (or $\bar{\pi}_{k}$ ) by $\hat{C}_{i j}$ (or $\hat{C}_{k}$ ) and $L_{i j}\left(\right.$ or $\left.L_{i j} \theta\right)$ by $\hat{C}_{w_{i j}}\left(\right.$ or $\left.\hat{C}_{w_{i j}}\right)$ in (4.3)-(4.6), with appropriate \pm 1 's. These are obtainable using the algorithm of $\S 2$. As an example, we will sketch the idea for SU $(4,1)$. All appropriate notation is contained in Figure 5. We must assume the reader is fully familiar with computations of the sort in [24, (5.3)], $\tau$-invariant calculations, etc. As a reference, we refer the reader to [9].

Begin with $\mathbf{J}_{\text {geo }}\left(C_{01}\right)=C_{w_{01}}$, by (2.26). Using $\tau$-invariant conditions, we find

$$
\begin{aligned}
& \mathbf{J}_{\text {geo }}\left(C_{02}\right)=a_{02} C_{w_{02}}+a_{31^{\theta}} C_{w_{31} \theta}, \\
& \mathbf{J}_{\text {geo }}\left(C_{03}\right)=a_{03} C_{w_{03}}+a_{22^{\ominus}} C_{w_{22} \theta}
\end{aligned}
$$

for some $a_{02}, a_{31^{\theta}}, a_{03}, a_{22^{\theta}} \in \mathbf{Z}\left[q^{1 / 2}, q^{-1 / 2}\right]$. (The point is, if $\alpha \in \tau(\bar{\pi}(\delta))$, then $\alpha$ is in the $\tau$-invariant of every irreducible constituent of $J(\bar{\pi}(\delta))$. An application of (2.26) gives a necessary condition for $C_{w}$ to occur in $J_{\text {geo }}\left(C_{\delta}\right)$. The $\tau$-invariant information is codified in Figure 5, as described in [3].) Next, computing with the Hecke algebra formulas of [24],

$$
\begin{aligned}
\left(T_{1}+1\right) \cdot \mathbf{J}_{\mathrm{geo}}\left(C_{02}\right) & =\left(T_{1}+1\right) \cdot a_{02} C_{w_{02}}+\left(T_{1}+1\right) \cdot a_{31^{\circ}} C_{w_{31} \theta} \\
& =a_{02} C_{w_{01}}+a_{02} q C_{w_{03}}+a_{31^{\theta}} C_{w_{31}} .
\end{aligned}
$$

On the other hand,

$$
\begin{aligned}
\mathbf{J}_{\text {geo }}\left(\left(T_{1}+1\right) \cdot C_{02}\right) & =\mathbf{J}_{\text {geo }}\left(C_{01}+q C_{03}\right) \\
& =\mathbf{J}_{\text {geo }}\left(C_{01}\right)+q \mathbf{J}_{\text {geo }}\left(C_{03}\right) \\
& =C_{w_{01}}+q a_{03} C_{w_{03}}+q a_{22^{\theta}} C_{w_{22} \theta}
\end{aligned}
$$

Combine (4.9), (4.10), and the fact that $\mathbf{J}_{\text {geo }}$ commutes with the Hecke algebra action. We conclude: $a_{02} \equiv 1, a_{03} \equiv 1$, and $a_{22^{\theta}} \equiv a_{31^{\theta}} \equiv 0$. This computes $\mathbf{J}_{\text {geo }}\left(C_{02}\right)$ and $\mathbf{J}_{\text {geo }}\left(C_{03}\right)$. Similar arguments easily lead to parts (i)-(iv) of $(4.4)$ (when $n=4$ ).

As a more interesting case, we apply $\tau$-invariant considerations to get

$$
\mathbf{J}_{\text {geo }}\left(C_{1}\right)=a C_{w_{04 \theta}}+b C_{w_{13 \theta}}+d C_{w_{03 \theta}},
$$

some $a, b, d \in \mathbf{Z}\left[q^{1 / 2}, q^{-1 / 2}\right]$. Because the sets $s \circ \delta$ in [24, (5.1)] are singletons in the $\mathbf{R}$-rank one case, the proof of (2.15) will insure the leading term coefficients $a \equiv b \equiv 1$. Next ,

$$
\begin{aligned}
\left(T_{4}+1\right) \cdot \mathbf{J}_{\mathrm{geo}}\left(C_{1}\right) & =\left(T_{4}+1\right) \cdot C_{w_{04 \theta} \theta}+\left(T_{4}+1\right) \cdot C_{w_{13 \theta}}+\left(T_{4}+1\right) \cdot d C_{w_{03 \theta} \theta} \\
& =C_{w_{04}}+(1+q) C_{w_{13} \theta}+d C_{w_{13 \theta}},
\end{aligned}
$$

and

$$
\mathbf{J}_{\mathrm{geo}}\left(\left(T_{4}+1\right) \cdot C_{1}\right)=\mathbf{J}_{\mathrm{geo}}\left(C_{0,4}\right)=C_{w_{04}} .
$$

Comparing terms, we get $d=-(1+q)$; i.e.,

$$
\begin{aligned}
\mathbf{J}_{\mathrm{geo}}\left(\hat{C}_{1}\right) & =\mathbf{J}_{\mathrm{geo}}\left(q^{-3} C_{1}\right)=q^{-3} C_{w_{04 \theta}}+q^{-3} C_{w_{13 \theta} \theta}-(1+q) q^{-3} C_{w_{038} \theta} \\
& =\hat{C}_{w_{04 \theta}}+\hat{C}_{w_{13 \theta} \theta}-(1+q) q^{-1 / 2} \hat{C}_{w_{038}} .
\end{aligned}
$$


This leads to weight filtration data:

$$
\begin{array}{ll}
\text { levels: } & -\hat{C}_{w_{03 \theta}}<\hat{C}_{w_{04 \theta}}+\hat{C}_{w_{13 \theta}}<-\hat{C}_{w_{13 \theta} \theta}, \\
\text { weights: } & q^{-1 / 2} q^{0} q^{1 / 2} .
\end{array}
$$

Following this same pattern of argument will succeed in all other R-rank one cases. Observe here, the key idea is that $\mathbf{J}_{\text {geo }}$ commutes with the $\mathscr{H}$ action! Details are left to the reader.

Proofs of (4.3)-(4.6): Socle PART. In view of (2.26), the characters of all of the $J(\bar{\pi}(\gamma))$ are now known. Our problem is to see that the pieces fit together as asserted. If $J(\bar{\pi}(\gamma))$ is irreducible or consists of two irreducible composition factors, then [6] handles this case. Assume $J(\bar{\pi}(\gamma))$ contains exactly three irreducible composition factors. We know this decomposition takes the form $J(\bar{\pi}(\gamma))=L_{w}+$ $L_{w}+L_{y}$, in the Grothendieck group. By self-duality $J(\bar{\pi}(\gamma))$ is either semisimple or has the asserted structure. If it were semisimple, $H_{0}(\bar{n}, J(\bar{\pi}(\gamma)))$ would have dimension three. Combining this with (1.5) and [7] yields a contradiction. Now, consider any of the remaining cases; these $J(\bar{\pi}(\gamma))$ have four composition factors. Precisely, we will have $J(\bar{\pi}(\gamma))=L_{w_{1}}+L_{w_{2}}+L_{y}+L_{y}$, in the Grothendieck group. To prove the assertion, it is enough (by self-duality) to show that neither $L_{w_{1}}$ nor $L_{w_{2}}$ are submodules of $J(\bar{\pi}(\gamma))$. This may be gleaned from the fact that all irreducible submodules of $J(\bar{\pi}(\gamma))$ define the same primitive ideal (see [35]).

It is perhaps worthwhile to comment on (1.4), in the case of higher R-rank. In this direction, we will describe two examples, each of which indicate the subtle (and interesting) structure of $J(\bar{\pi}(\gamma))$. Proofs are left to the reader, who may distill the necessary technical ideas from our above discussion.

The case of $\mathrm{PSP}_{2} \mathbf{R}$. For this subsection, $G_{\mathbf{R}}=\operatorname{PSP}_{2} \mathbf{R}$. We adhere to the notation of Vogan's article in [34], in particular, $A, B, \ldots, L$ parametrize the standard representations. In Figure 2, we include a picture of the $K$ - and $N$-orbit pictures, together with a "pedestrian description" of some of the attached irreducible representations. In (4.12), we have indicated the socle filtrations of $J(\bar{A}), \ldots, J(\bar{L})$. We have

(4.12) LemMA. If $G=\operatorname{PSP}_{2} \mathbf{R}$, then the weight filtrations of $J_{\text {geo }}(\bar{A}), \ldots, J_{\text {geo }}(\bar{L})$ correspond to the socle filtrations of $J(\bar{A}), \ldots, J(\bar{L})$, respectively. More precisely, we have

(i) $J(\bar{A})=J(\bar{D})=L_{2}, J(\bar{E})=J(\bar{G})=L_{21}, J(\bar{H})=J(\bar{J})=L_{212}, J(\bar{L})=L_{2121}$,

(ii) $J(\bar{B})=J(\bar{C})=L_{e} \oplus L_{e}<L_{2} \oplus L_{1} \oplus L_{1}<L_{e} \oplus L_{e}$,

(iii) $J(\bar{F})=L_{2}<L_{12} \oplus L_{12}<L_{2}$,

(iv) $J(\bar{I})=L_{21}<L_{121} \oplus L_{121} \oplus L_{1} \oplus L_{1}<L_{21}$,

(v) $J(\bar{K})=L_{212}<L_{2121} \oplus L_{12} \oplus L_{12}<L_{212}$.

As indicated in Figure 2, the modules with irreducible Jacquet modules correspond to constituents of continuations of holomorphic and antiholomorphic discrete series; a phenomena established in generality in [6]. Next, $H_{m, \mathbf{R}}$ is disconnected: $\left|H_{m} / H_{m}^{0}\right|=2$. For this reason, $J((\bar{\cdots}))$ carries an $M_{m}$-action, which is not detected 
by our discussions. More careful considerations allow one to detect these actions in the filtrations of (4.12). For example, for the large discrete series $[35] \bar{B}, J(\bar{B})$ has different actions on the $L_{1}$ components. (This fact was communicated to the second author, some time ago, by $\mathrm{H}$. Hecht.) These ideas will not be pursued here, but further indicate the delicate structure of the Jacquet modules.

The case of $\mathrm{SL}_{3} \mathrm{C}$. As a final example, fix $G_{\mathbf{R}}=\mathrm{SL}_{3} \mathrm{C}$. In Figure 3, we indicate the $K$ - and $N$-orbit structures. Here, we recall that the Dynkin diagram attached to $G_{\mathbf{R}}$ is of the form:
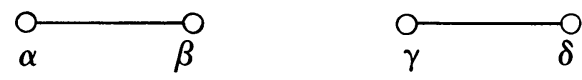

We let $W \times W$ act on the "basis" $W$ of $K\left(\mathscr{H}_{\mathscr{C}_{F}}\right)$, through the regular representation

$$
\left(w_{1}, w_{2}\right)(w, y)=\left(w, w_{1} y w_{2}^{-1}\right) .
$$

In the $\mathcal{O}_{F}^{\prime} \times \mathcal{O}_{F}^{\prime}$ category, coherent continuation is coordinatewise on the left. Consider the standard representation attached to $[121, e] \stackrel{\text { def }}{=} \delta_{\text {fund }}$. Then $\delta_{\text {fund }}$ is an irreducible fundamental series representation. Moreover, $\delta_{\text {fund }}$ is a large representation [35]. In Figure 4, we include the weight filtration of $J_{\text {geo }}\left(\delta_{\text {fund }}\right)$. This example is our first case of Loewy length $J_{\text {geo }}(\cdots)>3$. The reader is invited to find six "Verma submodules of $J_{\text {geo }}\left(\delta_{\text {fund }}\right)$." With some effort, this weight filtration may be shown to coincide with the socle filtration for $J\left(\delta_{\text {fund }}\right)$. Finally, notice that $J_{\text {geo }}\left(\delta_{\text {fund }}\right)$ has 44 composition factors, yet $G_{\mathbf{R}}$ only has $\mathbf{R}$-rank two; a sharp contrast to the fact that $J_{\text {geo }}(\bar{\pi}(\gamma))$ has at most four pieces in R-rank one!
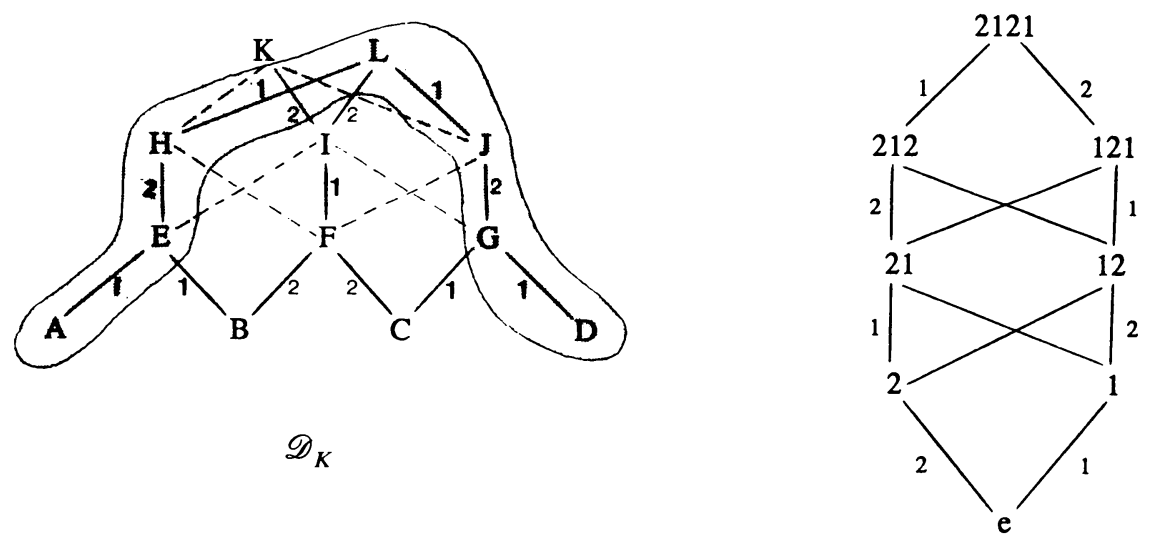

$\mathrm{L} \Leftrightarrow$ finite dimensional representation

$\mathrm{K} \Leftrightarrow$ largest growth, not finite dimensional

$\mathrm{A}, \mathrm{D} \Leftrightarrow$ holomorphic discrete series

$\mathrm{B}, \mathrm{C} \Leftrightarrow$ non holomorphic discrete series (large representations)

$2121 \Leftrightarrow$ finite dimensional

$\mathrm{e} \Leftrightarrow$ irreducible Verma module

FIGURE 2. Orbit pictures for $\mathrm{PSP}_{2} \mathbf{R}$ 

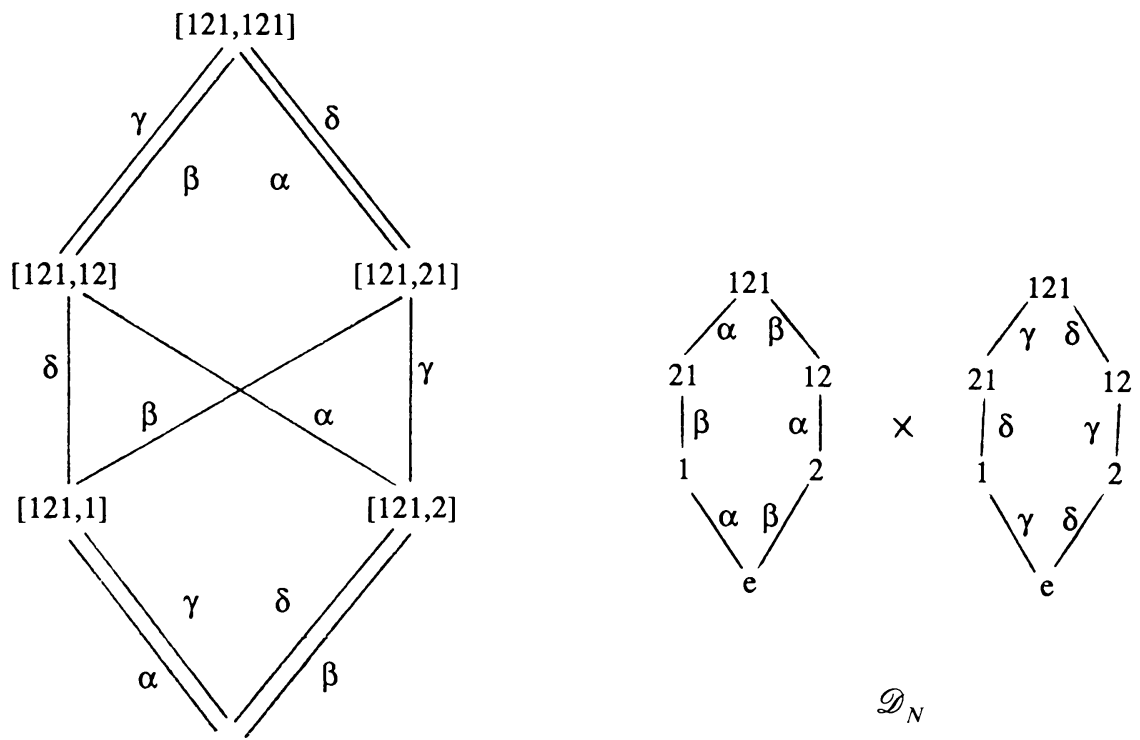

$[121, \mathrm{e}]$

$\mathscr{D}_{K}$

$[121, \mathrm{e}]=\delta_{\mathrm{f}}=$ fundamental series representation

$[121,121]=$ standard representation with finite dimensional quotient

FiguRE 3. Orbit pictures for $\mathrm{SL}_{3} \mathrm{C}$

weights

\begin{tabular}{|c|c|}
\hline \multirow{7}{*}{$\mathrm{J}_{\mathrm{geo}}\left(\delta_{\text {fund }}\right)=$} & $\mathrm{L}_{(\mathrm{e}, \mathrm{e})}$ \\
\hline & $\mathrm{L}_{(2, e)} \oplus \mathrm{L}_{(\mathrm{e}, 2)} \oplus \mathrm{L}_{(1, e)} \oplus \mathrm{L}_{(e, 1)}$ \\
\hline & 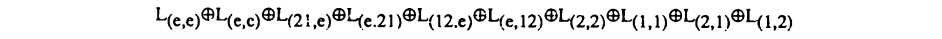 \\
\hline & $\mathrm{L}_{(121, e)} \mathrm{L}_{(\mathrm{e}, 121)} \oplus \mathrm{L}_{(21,1)} \mathrm{L}_{(12,2)} \mathrm{L}_{(1,21)} \mathrm{L}_{(2,12)} \oplus \mathrm{L}_{(2, e)} \oplus \mathrm{L}_{(2, e)} \oplus \mathrm{L}_{(e, 2)} \oplus \mathrm{L}_{(e, 2)} \oplus \mathrm{L}_{(1, e)} \oplus \mathrm{L}_{(1, e)} \oplus \mathrm{L}_{(e, 1)} \oplus \mathrm{L}_{(e, 1)}$ \\
\hline & 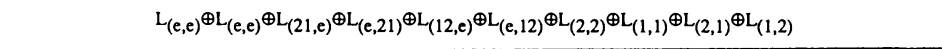 \\
\hline & $\mathrm{L}_{(2, \mathrm{e})} \oplus \mathrm{L}_{(\mathrm{e}, 2)} \oplus \mathrm{L}_{(1, \mathrm{e})} \oplus \mathrm{L}_{(\mathrm{e}, 1)}$ \\
\hline & $\mathrm{L}_{(\mathrm{e}, \mathrm{e})}$ \\
\hline
\end{tabular}

FIGURE 4. The weight filtration for $\mathbf{J}_{\text {geo }}\left(\delta_{\text {fund }}\right)$

5. A closing remark. Our fundamental interest in the structure of Jacquet modules stems from the isomorphism in (1.5): Understanding the $\bar{n}$-homology of $\bar{\pi}(\gamma)$ can be transferred to the problem of computing $H_{*}(\bar{n}, J(\bar{\pi}(\gamma)))$. Since $J(\bar{\pi}(\gamma))$ is in category $\mathcal{O}_{F, m}^{\prime}$, it is a "highest weight type module" and there is hope of extracting $\bar{n}$-homology information from the knowledge of its socle filtration. (If $J(\bar{\pi}(\gamma))$ is 
irreducible, i.e., $J(\bar{\pi}(\gamma))=L_{w}$, some $w \in W$, then the Kazhdan-Lusztig conjectures for $g$ completely solve the $\bar{n}$-homology problem; cf. [6].) The amazing fact is that this naive philosophy succeeds in the case of $\mathbf{R}$-rank one. These details will appear elsewhere, but we will indicate an example, which illustrates the subtle nature of the problem.

Fix $G_{\mathbf{R}}=\operatorname{SU}(4,1)$ and refer to Figure 5 for the structure of $\mathscr{D}_{K}$ and $\mathscr{D}_{N, m}$. Also recall our remarks in $\S 4$. We wish to describe $H_{*}\left(\bar{n}_{1}, \bar{\pi}_{2}\right)$. Here, $\bar{\pi}_{2}$ (see Figure 5) is a nonholomorphic discrete series representation. Recalling our minimal parabolic $\mathfrak{p}_{m}=\mathfrak{m}_{m} \oplus \mathfrak{a}_{m} \oplus \mathfrak{n}_{m}$, we content ourselves to compute $H_{*}\left(\bar{n}_{m}, \bar{\pi}_{2}\right)$. Since these homology groups are semisimple $\mathfrak{m}_{m} \oplus \mathfrak{a}_{m}$-modules, Kostant's theorem and a collapsing spectral sequence leads to $H_{*}\left(\bar{n}, \bar{\pi}_{2}\right)$, in the standard way. We find, as an $\mathfrak{m}_{m} \oplus \mathfrak{a}_{m}$-module,

$$
H_{k}\left(\overline{\mathrm{n}}_{m}, \bar{\pi}_{2}\right)= \begin{cases}\sigma_{13^{\theta}} \oplus \sigma_{12^{\theta}} \oplus \sigma_{22^{\theta}}, & k=0, \\ \sigma_{03^{\theta}} \oplus \sigma_{12^{\theta}} \oplus \sigma_{21^{\theta}} \oplus \sigma_{02^{\theta}} \oplus \sigma_{11^{\theta}}, & k=1, \\ \sigma_{02^{\theta}} \oplus \sigma_{01^{\theta}} \oplus \sigma_{11^{\theta}}, & k=2, \\ \sigma_{01^{\theta}}, & k=3, \\ 0, & k \geqslant 4 .\end{cases}
$$

length

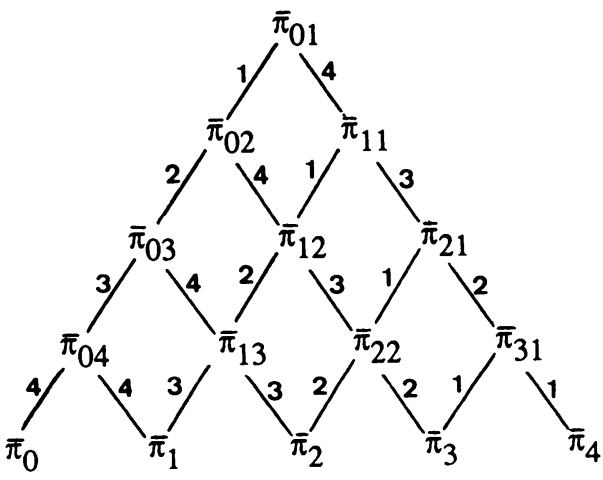

K-orbits on $\mathscr{B}$

$\bar{\pi}_{01}=$ finite dimensional

$\bar{\pi}_{0}, \bar{\pi}_{4}=$ holomorphic and antiholomorphic discrete series

$\bar{\pi}_{1}, \bar{\pi}_{2}, \bar{\pi}_{3}=$ nonholomorphic discrete series

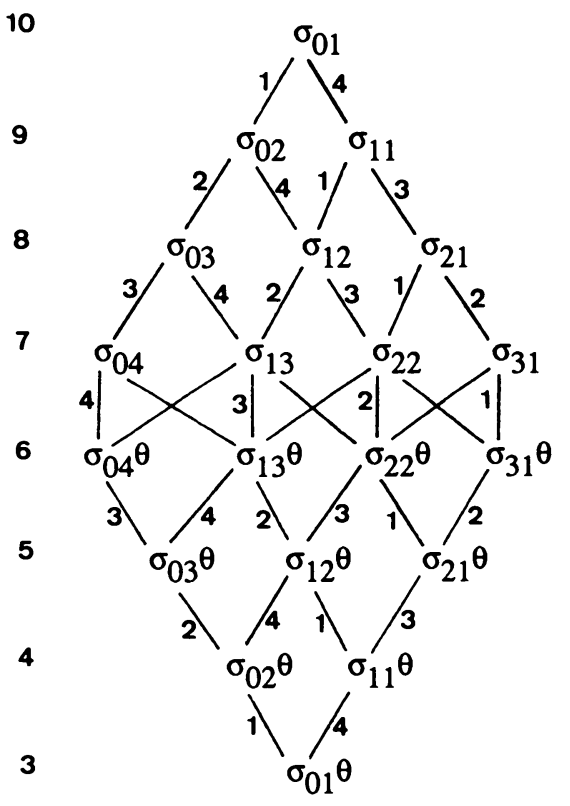

$\mathrm{P}_{\mathrm{m}}$-orbits on $\mathscr{B}$

FIGURE 5. Orbit structures for SU(4,1) 
One can carry out similar calculations for all $\bar{\pi}_{i j}, \bar{\pi}_{k}$ for all $G_{\mathbf{R}}$ as considered in (4.1). The main point is to analyze the socle filtrations of the Jacquet modules and use the structure theory of the generalized Verma modules in $[\mathbf{3}, \mathbf{2 0}, \mathbf{2 1}]$. The problem is tractable, because the Loewy length of $J(\bar{\pi}(\gamma))$ and $V_{w_{t}}$ (the generalized Verma modules) are bounded, independent of $G_{\mathbf{R}}$ in (4.1). Details appear in [9].

We close with a subtle observation. Consider (5.1) above and form the Osborne character:

$$
\begin{aligned}
\Delta \Theta\left(\bar{\pi}_{2}\right)= & \sigma_{13^{\theta}}+\sigma_{22^{\theta}}+\sigma_{12^{\theta}}-\left(\sigma_{03^{\theta}}+\sigma_{12^{\theta}}+\sigma_{21^{\theta}}+\sigma_{02^{\theta}}-\sigma_{11^{\theta}}\right) \\
& +\left(\sigma_{02^{\theta}}+\sigma_{11^{\theta}}+\sigma_{01^{\theta}}\right)-\sigma_{01^{\theta}} \\
= & \sigma_{13^{\theta}}+\sigma_{22^{\theta}}-\sigma_{03^{\theta}}-\sigma_{21^{\theta}} .
\end{aligned}
$$

This example dramatically illustrates the massive cancellation inherent in the "Euler

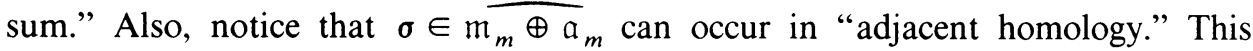
"nonvanishing" result is in sharp contrast to the Verma situation. For other " $n$ ' $s$ ", Vogan was able to establish a vanishing condition, precluding such phenomena and leading to the computation of those " $n$ "-homology groups [31].

Note ADDED IN PROOF. (i) If $G$ has connected Cartan subgroups, conjecture (1.4) is true. In particular, $\mathbf{J}_{\text {geo }}$ gives a $D$-module construction of the Jacquet functor, in the weaker sense of (1.4).

(ii) For general $G$, a variation of (1.4) is established in [38].

Namely, we establish the existence of a computable g-filtration on $J(\bar{\pi}(\gamma))$ with semisimple subquotients. For a precise discussion we refer the reader to [38] and especially [38, (1.23)].

\section{REFERENCES}

1. A. Beilinson and I. Bernstein, Localisation de g-modules, C. R. Acad. Sci. Paris Sér. I 292 (1981), $15-18$.

2. A. Beilinson, J. Bernstein and P. Deligne, Analyse et topologie sur les espaces singuliers. I, Astérisque 100, Soc. Math. France, 1982.

3. B. Boe and D. Ćollingwood, Comparison theory for the structure of induced representations. II, Math. Z. 190 (1985), 1-11.

4. J. Brylinski, Homologie d'intersection et faisceaux pervers, Séminaire Bourbaki, 34e année, no. 585 , $1981-82$.

5. W. Casselman, Jacquet modules for real reductive groups, Proc. Internat. Congr. Math., Helsinki, 1978, pp. 557-563.

6. D. Collingwood, Harish-Chandra modules with the unique embedding property, Trans. Amer. Math. Soc. 281 (1984), 1-48.

7. Embeddings of Harish-Chandra modules, n-homology and the composition series problem: the case of real rank one, Trans. Amer. Math. Soc. 285 (1984), 565-579.

8. The n-homology of Harish-Chandra modules: generalizing a theorem of Kostant, Math. Ann. 272 (1985), 161-187.

9. __ Representations of real rank one Lie groups, Pitman Research Notes in Math., No. 137, Pitman, London, 1985.

10. P. Deligne, La conjecture de Weil. II, Inst. Hautes Étude Sci. Publ. Math., no. 52, 1980.

11. O. Gabber and A. Joseph, On the Bernstein-Gelfand-Gelfand resolution and the Duflo sum formula, Comp. Math. 43 (1981), 107-131.

12. M. Goresky and R. MacPherson, Intersection homology. II, Invent. Math. 72 (1983), 77-79.

13. A. Grothendieck et al., (SGA 4) Théorie des topos et cohomologie étale des schémas, Lecture Notes in Math., vols. 269, 270, 305, Springer-Verlag, Berlin and New York, 1972-1973, pp. 270-305.

14. , (SGA 4 1/2) Cohomologie etale, Lecture Notes in Math., vol. 569, Springer-Verlag, Berlin and New York, 1977. 
15. , (SGA 7) Groupes de monodromie en géométrie algébrique, Lecture Notes in Math., vol. 288,

Springer-Verlag, Berlin and New York, 1972; Part II in vol. 340, 1973.

16. $\mathrm{H}$. Hecht, On the characters and asymptotics of representations of a real reductive Lie group, Math. Ann. 242 (1979), 103-126.

17. H. Hecht and Schmid, Characters, asymptotics, and n-homology of Harish-Chandra modules, Acta Math. 151 (1983), 49-151.

18. , On the asymptotics of Harish-Chandra modules, J. Reine Angew. Math. 343 (1983), 169-183.

19. S. Helgason, Differential geometry, Lie groups and symmetric spaces, Academic Press, New York, 1978.

20. R. Irving, Projective modules in the category $\mathcal{O}_{s}$ : Self-duality, Trans. Amer. Math. Soc. 292 (1986), 701-732.

21. __ Projective modules in the category $\mathcal{O}_{s}$ : Loewy series, Trans. Amer. Math. Soc. 291 (1986), $733-754$.

22. M. Kashiwara, The Riemann-Hilbert problem for holonomic systems, Publ. Res. Inst. Math. Sci. $\mathbf{4 3 7}$ (1983).

23. D. Kazhdan and G. Lusztig, Schubert varieties and Poincaré duality, Proc. Sympos. Pure Math., vol. 36, Amer. Math. Soc., Providence, R. I., 1980, pp. 185-203.

24. G. Lusztig and D. Vogan, Singularities of closures of K-orbits on flag manifolds, Invent. Math. 71 (1983), 365-370.

25. A. Mebkout, Sur le probleme de Hilbert-Riemann, Lecture Notes in Phys., vol. 126, Springer-Verlag, Berlin and New York, 1980, pp. 99-110.

26. D. Miličić, Notes on localisation of modules over enveloping algebras, handwritten manuscript, 1983.

27. J. Milne, Étale cohomology, Princeton Univ. Press, Princeton, N.J , 1980.

28. W. Schmid, Vanishing theorems for Lie algebra cohomology and the cohomology of discrete subgroups of semisimple Lie groups, Adv. in Math. 41 (1981), 78-113.

29. W. Silva, The embeddings of the discrete series in the principal series for semisimple Lie groups of real rank one, Trans. Amer. Math. Soc. 261 (1980), 303-368.

30. D. Vogan, Irreducible characters of semisimple Lie groups. I, Duke Math. J. 46 (1979), 61-108.

31. Irreducible characters of semisimple Lie groups. II: The Kazhdan-Lusztig conjectures, Duke Math. J. 46 (1970), 805-859.

32. __ Irreducible characters of semisimple Lie groups. III: Proof of the Kazhdan-Lusztig conjectures in the integral case, Invent. Math. 71 (1983), 381-417.

33. _ Irreducible characters of semisimple Lie groups. IV: Character multiplicity duality, Duke Math. J. 49 (1982), 943-1073.

34. The Kazhdan-Lusztig conjecture for real reductive groups, Representation Theory of Reductive Lie Groups, Proc. 1982 Park City Conference, Progress in Math., Birkhäuser, Boston, 1983.

35. __ Gelfand-Kirillov dimension for Harish-Chandra modules, Invent. Math. 48 (1978), 75-98.

36. __ Representations of real reductive Lie groups, Progress in Math., Birkhäuser, Boston, 1981.

37. N. Wallach, Representations of semisimple Lie groups and Lie algebras, Queen's Papers in Pure and Appl. Math. 48 (1977), 154-247.

38. L. Casian and D. Collingwood, Complex geometry and the asymptotics of Harish-Chandra modules for real reductive Lie groups. II, Invent. Math. 86 (1986), 255-286.

39. Complex geometry and the asymptotics of Harish-Chandra modules for real reductive Lie groups. III: Estimates on n-homology, preprint, 1985.

Department of Mathematics, The University of Utah, Salt Lake City, Utah 84112 (L. G. Casian and D. H. Collingwood)

Department of Mathematics, The University of Oregon, Eugene, Oregon 97403 (D. H. Collingwood) 